Caridade, S., \& Braga, T. (2020). Youth cyber dating abuse: A meta-analysis of risk and protective factors. Cyberpsychology: Journal of Psychosocial Research on Cyberspace, 14(3), Article 2. https://doi.org/10.5817/CP20203-2

\title{
Youth Cyber Dating Abuse: A Meta-Analysis of Risk and Protective
} Factors

\author{
Sónia Caridade ${ }^{1,2,3} \&$ Teresa Braga ${ }^{4}$ \\ ${ }^{1}$ Human and Social Sciences Faculty (FCHS-UFP), University Fernando Pessoa (UFP), Oporto, Portugal \\ 2 Interdisciplinary Center for Gender Studies (CIEG), Higher Institute of Social and Political Sciences of the University of Lisbon \\ (ISCSP-UL), Lisboa, Portugal \\ ${ }^{3}$ Observatory Violence and Crime (OPVC), University Fernando Pessoa (UFP), Oporto, Portugal \\ ${ }^{4}$ Integration and Probation Penitentiary Services, Aisne, France
}

\begin{abstract}
Cyber Dating Abuse (CDA) has been identified as a prevalent and negative experience for youth, which highlights the need to identify the factors associated with the occurrence of this phenomenon. Thus, this meta-analysis aims to determine the factors associated with youth CDA perpetration and victimization, identifying which of the factors present the strongest risk and protective effects. We identified 16 studies and 17 independent samples, including a total sample of 12,760 adolescents and young adults. Results showed that individual and intimate relation factors were related to both CDA perpetration and victimization, and peer factors were significantly associated with CDA perpetration; individual sociodemographic factors were unrelated to CDA. Findings from this metaanalysis provide valuable evidence to develop CDA prevention and intervention strategies.
\end{abstract}

Keywords: Cyber dating abuse; youth; risk factors; protective factors; meta-analysis

\section{Introduction}

Digital practices and networking through a wide variety of communication tools (e.g., text messages, emails, video calls) have proliferated in the last decades, introducing important changes in adolescents and young people's social interactions, including those involving the development and maintenance of dating relationships (Burke et al., 2011). On one hand, digital practices can improve adolescents' socialization process, such as the ability to exercise self-control, the promotion of tolerance and respect for others and critical thinking, and the ability to make decisions, but on the other hand, they can also become an avenue of increased risk of victimization and perpetration of abusive behaviors (Hellevik, 2019; Pujazon-Zazik \& Park, 2010). In the last few years, several abusive interactions through technology have been identified, including Cyber Dating Abuse (CDA; Borrajo, Gámez-Guadiz, Pereda et al., 2015; Zweig et al., 2013), defined as a form of control and harassment by the dating partner through the use of technologies and the media (Zweig et al., 2014). CDA can involve multiple abusive behaviors through digital interactions, such as daily control and surveillance of the dating partner, sending/posting offensive or humiliating comments, photos or videos to/of the dating partner, and sending emails or messages containing different threats (e.g., Bennett et al., 2011; Burke et al., 2011; Zweig et al., 2013). A recent review (Gámez-Guadix et al., 2018) showed that these behaviors may integrate different typologies of abuse such as: cyber psychological control or monitoring (e.g., the need to know, at all times, the whereabouts of the dating partner and with whom he/she is), cyber harassment (e.g., repeated and insidious calls), and cyber psychological and verbal aggression (e.g., insults, threats, and humiliations). Other studies (e.g., Watkins et al., 2018) have delineated typologies of CDA differently (i.e., psychological cyber aggression, sexual cyber aggression, and stalking 
cyber aggression) Together, these studies illustrate an ongoing effort to establish meaningful typologies of CDA. Research has revealed worrying figures of youth CDA victimization and perpetration. In a review of 12 studies concerning technology-assisted adolescent dating abuse, Stonard et al. (2014) found victimization and perpetration rates of around 55\%. A critical review of digital dating abuse measures (Brown \& Hegarty, 2018) found perpetration rates as high as $91 \%$ among youth. Likewise, in a systematic review of 44 studies on youth CDA (Caridade et al., 2019), the maximum victimization and perpetration rates exceeded $90 \%(92.0 \%$ and $93.7 \%$, respectively). However, these same reviews also documented CDA prevalence rates as low as 5.8\% for CDA victimization (Caridade et al., 2019) and 6\% for CDA perpetration (Brown \& Hegarty, 2018) concluding, as other studies (e.g., Flach \& Deslandes, 2017; Stonard et al., 2014), that research on CDA has produced extremely variable and difficult to interpret results. The lack of consensus among the terms [e.g., Cyber dating Abuse, Digital Dating Abuse, Electronic Aggression, Electronic Dating Aggression, and Intimate Partner Cyber Aggression], the operationalization and among the tools used to measure CDA, as well as methodological characteristics of the studies (e.g., sample size, sampling context, time interval considered) were suggested as explanatory factors of these variable prevalence rates (Brown \& Hegarty, 2018; Caridade et al., 2019; Flach \& Deslandes, 2017; GámezGuadix et al., 2018; Stonard et al., 2014).

Studies on CDA have revealed associations between experiencing CDA and negative implications for victims. Van Ouytsel, Ponnet, Walrave, and Temple (2016) concluded that adolescent victims of CDA presented an increased risk of involvement in health risk behaviors, such as alcohol consumption. A cross-sectional study (Zweig et al., 2014), developed among a large sample of youth $(n=3,745)$, found that CDA is related to several emotional problems, such as depressive symptoms, feelings of hostility and anger, and anxiety disorders. In turn, Lu et al. (2018) analyzed both the cross-sectional and longitudinal associations between CDA victimization and mental health (i.e., anxiety, PTSD, and depression), and substance use (i.e., alcohol, cigarettes, marijuana, and hard drugs), and they concluded that CDA victimization was cross-sectionally but not longitudinally associated with measures of mental health and substance use. Thus, CDA has been associated with negative implications for the victims, but these might manifest themselves more acutely and not over extended periods of time. The negative implications associated with CDA victimization, as well as the high prevalence rates of CDA, found by several studies, highlight the urgency to prevent and intervene in this phenomenon.

\section{Risk and Protective Factors of Cyber Dating Abuse}

Risk and protective factors are considered key elements when aiming to prevent the (re)occurrence of abusive behaviors, such as those including in CDA. Indeed, these factors have been defined as those associated with, respectively, an increase and a decrease in the probability of the occurrence of problem outcomes (Farrington et al., 2016). Several explanatory theories of youth CDA have been advanced, highlighting different risk and protective factors. For instance, the social learning theory defends that aggressive behaviors are learned through different social interactions with, for example, family and peers (Bandura, 1977, as cited by Villora et al., 2019). The lifestyleroutine activities theory argues that engaging in online risk behaviors is the most important predictor of becoming a victim of digital controlling behavior by an intimate partner (Reyns et al., 2011). A review of theories commonly used in the social sciences literature to explain dating violence (DV) perpetration has defended, however, the importance of using integrative theories (e.g., Dardis et al., 2015). In this sense, socio-ecological theories have been identified as a useful theoretical framework to understand and grasp the overall factors that are related with CDA (Zweig et al., 2014). This approach focuses not only on the individual, but on the importance of the contexts of the youths' life and, in this sense, identifies multiple levels of the social ecology (e.g., individual characteristics; family dynamics; relationships with peers; school performance) that interact with the developmental path of adolescents, influencing their behavior and experiences (Bronfenbrenner \& Morris 1998).

While ecological models have gain support among research on youth CDA (e.g., Peskin et al., 2017; Reed et al., 2018; Smith-Darden et al., 2017; Zweig et al., 2014), possibly due to their greater comprehensiveness, empirical studies still seem to focus on particular levels of the social ecology (e.g., individual, intimate relations) while neglecting others (e.g., family, community). The same can be said for risk and protective factors, as the first factors have been the main focus of studies in this domain. Specifically, according to a systematic review aimed to describe the main objectives and findings of studies on youth CDA, Caridade et al. (2019) concluded many studies analyzed variables related to CDA but they focused mainly on individual risk factors. The few studies that included protective factors, such as parental involvement (e.g., Peskin et al., 2017; Smith-Darden et al., 2017), parent communication 
and parent child-closeness (e.g., Peskin et al., 2017) and high family cohesion (Rivas \& Gimeno, 2017), concluded that these are indeed related to CDA. Furthermore, with regard to individual risk factors, Caridade et al. (2019) identified a long list of variables related to youth CDA perpetration (e.g., legitimization of CDA, behavioral or romantic jealousy, belief in myths about love, sexist beliefs and endorsement of gender stereotypes, narcissism vulnerability and grandiosity, bullying and cyberbullying, risk behaviors) and CDA victimization (e.g., risk behaviors, initiated sexual activity, time spend on social networking, depressive symptoms, anxiety, emotional/psychological distress, anger/hostility, lower self-esteem, narcissism vulnerability and grandiosity), revealing a disperse overall picture of which particular factors are (more) related with youth CDA.

The disperse picture of individual variables associated with youth CDA is further complicated by inconsistent findings, found particularly among sociodemographic individual variables. Zweig et al. (2013) found that female students were more often victims of cyber sexual abuse than male, but they were less often victims of non-sexual cyber abuse. In Deans and Bhogal's (2017) study, gender was significantly associated with CDA, with women reporting less perpetration of CDA than men, contrary to other previous studies (e.g., Bennett et al., 2011; Burke et al., 2011) were women were more likely than men to perpetrate controlling online behaviors. These inconsistent findings have been attributed to the fact that men and women perpetrated CDA differently: men tend to perpetrate more direct acts of aggression (Perry \& Pauletti 2011; Taylor \& Xia, 2018) while women more indirect acts (Hyde, 2005), such as monitoring behaviors (Taylor \& Xia, 2018). Studies (e.g., Sánchez et al., 2015; Smith et al., 2018) examining the relation between age and CDA victimization have also reached discrepant results: Smith et al. (2018) concluded that age was not associated with cyber victimization, cyber perpetration, or mutual cyber violence; in turn, Sánchez et al. (2015) reveled a positive association between age and CDA victimization, although the size of these associations varied according to the different abusive typologies. These inconsistent results, indicating that sociodemographic variables are positively, negatively or not related to CDA, do not allow their characterization as being risk or protective factors (or neither) and need further exploration and scientific clarification.

Individual sociodemographic variables have also been identified as moderators of the relation between CDA and associated variables. For example, with regard to CDA victimization, girls were more likely to revealed negative emotions (Reed et al., 2016) and to anticipate more distress from sexting compared to boys (Bennett et al., 2011; Smith et al., 2018). In turn, boys who had experienced CDA engaged in more risk behaviors (alcohol consumption and unprotected sexual behavior) than girls (Van Ouytsel, Ponnet, Walrave, \& Temple, 2016). Likewise, a greater legitimization of CDA (Borrajo et al., 2015b) has been related to CDA perpetration specially among boys. In addition, age has been identified as a moderator of the relationship between beliefs in myths about love and perpetration of CDA, with this relationship being statistically significant among younger but not older youth. Younger adolescents tend to present more traditional and unrealistic visions of intimate relations, have less experience in relationships and are less aware of how their beliefs may affect their abusive behaviors (Borrajo et al., 2015b).

At last, it is also important to consider the different contexts in which dating relationships take place (SeiffgeKrenke \& Connolly, 2010), in order to understand how protective and risk factors may operate differently according to the culture. Despite the recentness of research in the field of CDA, some studies (e.g., Gámez-Guadix et al., 2014; Jiménez et al., 2017) have sought to analyze the impact of culture on CDA. For instance, Jiménez et al. (2017) analyzed the cultural invariance of an instrument assessing online control, intrusiveness and jealousy among 530 adolescents living in Spain and in Mexico. They concluded that jealousy, control and intrusiveness were similar across the youth from these countries. Nevertheless, the authors also found that Mexicans youth, especially boys compared to girls, presented higher levels of control and intrusiveness, explaining these results by the higher levels of sexism and gender inequality found in the Mexican culture. This line of research should be encouraged in order to inform culturally adapted intervention efforts.

\section{The Current Study}

Identifying factors that are related with youth CDA is essential for the development of effective prevention and intervention programs. Recent studies have grasped this task, but results are dispersed and inconsistent, lacking of cohesion and systemization. The existing efforts in systematizing knowledge on youth CDA (Brown \& Hegarty, 2018; Flach \& Deslandes, 2017; Gámez-Guadix et al., 2018; Stonard et al., 2014; Taylor \& Xia, 2018) have focused 
mainly on the nature, prevalence, and impact of CDA. We aimed precisely to fill these gaps in the literature by conducting a meta-analysis of the risk and protective factors of youth CDA. Meta-analyses have been recognized as an excellent opportunity to summarize available knowledge, identify variables that are related to the phenomenon, and thus better define the priorities for intervention and research (Hébert et al., 2017). Thus, with the present meta-analysis we intended to answer the following questions: Which factors risk and protective factors have been associated with youth CDA? Which risk and protective factors are (more strongly) associated with youth $\mathrm{CDA}$ ? Are the relations between risk and protective factors and youth CDA moderated by the sociodemographic characteristics of the participants, namely sex and age? Additionally, does the methodological quality of the studies moderate the factors-youth CDA relations?

\section{Method}

The present meta-analysis was conducted according to the Preferred Reporting Items for Systematic Reviews and Meta-Analyses (PRISMA) guidelines (Moher et al., 2009) as well as the Meta-Analysis Reporting Standards (MARS) recommendations (APA Publications and Communications Board Working Group on Journal Article Reporting Standards, 2008).

\section{Eligibility Criteria}

\section{Participants}

To be included, studies had to present results on adolescents and/or young adults, i.e., the sample's mean age was between 10 to 26 years old, inclusive, according to APA (n.d., 2002) definitions of adolescence and young adults. We also included studies that characterized the participants as being, for instance, "adolescents", "students", "youth" "young adults", and/or "emerging adults", but did not specify their mean age.

\section{Independent Variables}

In line with an socio-ecologic approach for studying causes and correlates (Bronfenbrenner \& Morris 1998), the current meta-analysis focused on all possible risk and protective factors. Thus, the factors associated with youth CDA could be from the individual, family, peer, school, and the larger community domain. In addition, they could be conceptualized as either potential risk factors (i.e., associated with a higher likelihood of problem outcomes), protective factors (i.e., associated with a lower likelihood of problem outcomes), or control variables (i.e., frequently the case of sociodemographic variables).

\section{Dependent Variables}

Studies were included if they assessed the occurrence of any form of violence through the use of technologies and the media occurring exclusively in an intimate dating relationship - cyber dating abuse. Both CDA victimization and perpetration were included, but these had to be assessed separately in the studies, as they were considered different variables.

\section{Study Type and Design}

Given the meta-analytic nature of the present study, we focused on quantitative or mixed research. No restrictions were placed on the study's design, namely using a longitudinal design (despite being more appropriate given our objectives), because this criterion would have resulted in the exclusion of the vast majority of the studies. In order to establish the direction of the relation between the potential risk and protective factors and CDA, we only included studies that performed predictive analyses and treated CDA as an outcome.

\section{Search and Selection Strategies}

First, we defined the different keywords and their combination in order to create the search equation as followed: ("Youth" OR "Adolescents" OR "Students") AND ("Cyber dating" OR "Digital Dating" OR "Partner Cyber") AND ("Abuse" OR 
"Violence" OR "Aggression"). This combination was then used to search several electronic databases, namely: Academic Research Complete; Business Source Complete; Complementary Index; ERIC/EBSCOhost; Psychology and Behavioral Sciences Collection; PubMed; Science Direct; Scopus, and Social Sciences Citation Index. We limited our search to titles, abstracts, or keywords and to manuscripts written in English, Portuguese, or Spanish. No other restrictions were placed, such as date of publication or type of manuscript (e.g., article, conference paper). This database search was conducted until February 2019. In addition, we examined the reference lists of review articles addressing CDA (Brown \& Hegarty, 2018; Gámez-Guadix et al., 2018; Flach \& Deslandes, 2017) in order to identify relevant studies not yet included through the databases search. Finally, we contacted authors in the field of dating abuse and CDA to request for additional references.

We screened the titles and abstracts of the studies in order to decide if they could eventually meet our eligibility criteria. If so, full-texts were then retrieved and fully read to reach a final decision.

\section{Independence of Study Samples}

A key issue in conducting meta-analyses is ensuring the independence of study results, that is, that no particular sample is duplicated and thus weighted more strongly compared to others (Lipsey \& Wilson 2001). We handled this issue by establishing and using the subsequent four criteria: 1) we chose the longest time span between the associated variables and CDA, when studies assessed these variables at multiple points in time among the same sample; 2) we favored analyses that included the largest number of associated variables in detriment to those that focused only on the significant ones, when both were presented for the same sample; 3) when studies assessed both overall and subtypes of CDA (e.g., aggression, control, sexting), we preferred the overall measure as there was insufficient data to meta-analyze CDA by subtypes. In the same sense, when studies presented results exclusively on CDA by subtypes, we opted to average them into a global measure of CDA; 4) whenever presented in the studies, we coded effect sizes per sex, and treated males and females as independent samples.

\section{Coding Procedures}

A codebook was developed to gather information on each of the included studies, including the following key characteristics: reference information (authors, year publication); study location (geographic location); sampling characteristics (sampling frame); samples' characteristics ( $n$, age, sex, ethnicity/race); associated variables (name, categories of belonging), and CDA (perpetration or victimization, assessment measure) and effect size data. With regard to the variables' categories of belonging, these were first classified as "risk" or "protective factors" depending on their theoretical relation to CDA (i.e., those associated with, respectively, an increase or a decrease in the probability of the occurrence of problem outcomes; Farrington et al., 2016). For example, family conflict was classified as a risk factor and family closeness was classified as a protective factor, as the first was conceptualized as possibly increasing the probability of occurrence of CDA and the last as possibly decreasing the occurrence. We decided not to classify sociodemographic factors as risk or protective bearing in mind the contrary findings regarding the risk, protective or inexistent effects of these variables on youth CDA. Sociodemographic, risk and protective factors were further classified into upper and lower case categories, developed based on the CDA literature (Peskin et al., 2017). Specifically, factors were coded as: i) Individual - factors that are related with the persons functioning and/or experiences such as Adverse experiences, Behavioral, Mental and Physical Health, or Psychosocial; ii) Relational - factors that involve Intimate relations, Peer, or Family; iii) and Community - factors associated with School or Neighborhood. Table 1 and Table 2 include examples of the variables included in these upper and lower case categories. Caution was taken in guaranteeing that similar variables across studies were assessed in the same direction (e.g., lower frequency values indicating a lower presence of the construct).

The codebook also included an evaluation of studies' methodological quality based on the Cambridge Quality Checklist for risk factors (Murray et al., 2009). In line with our aims, we included the checklist's five items to identify high-quality studies of correlates - adequate sampling method (e.g., random sampling), adequate response rates (response rates $\geq 70 \%$ ), adequate sample size (sample size $\geq 400$ ), good measure of correlate (e.g., reliability coefficient $\geq .75$ ), and good measure of outcome (e.g., reliability coefficient $\geq .75$ ) - and the additional study designs item - cross-sectional data, retrospective data, or prospective data - for studies of risk factors (conceptualized as correlates that precede the outcome). For each of the first five items, studies are scored ' 1 ' if the feature is present or ' 0 ' if the feature is not present or unknown. The study design item is coded from ' 1 ' to ' 3 '. The six scores were 
summed to give a total score out of 8 , with higher scores reflecting more confidence that accurate conclusions can be drawn about correlates and risk factors.

All articles were independently coded by the two authors, and disagreements were resolved through discussion. The interrater agreement was found to be a mean Kappa of .79, 95\% $\mathrm{Cl}[.41, .95]$ and a mean intraclass correlation coefficient of .96, 95\% Cl [.92, .99].

\section{Data Analysis}

Analyses were conducted using the statistical software Comprehensive Meta-Analysis (version 2). The correlation coefficient ( $r$ ) was used as the common metric. When individual studies did not provide an $r$, other statistics (e.g., odds ratio, $t$-values) were converted into this metric using the statistical software or, when this was not possible, using conversion formulas (Cohen, 1988; Rosenthal, 1994). Specifically, regression coefficients $(B$ and $\beta$ ) were converted into $t$-values by dividing them by their standard error (Stanley \& Jarrell, 1989) and then converted into an $r$. Standardized regression coefficients $(\beta)$ reported without their standard error were converted into an $r$ using Peterson and Brown's (2005) approximation formula.

For the estimation of the global effects, the effect sizes were converted into Fisher's $Z$ scores, as recommended by Borenstein et al. (2009), because the distribution of this metric is normal, thus allowing for more precise statistical tests. The estimates were then converted back to correlations to ease interpretation. Cohen's guidelines were used to interpret the magnitude of the effect: .10 is considered small, .30 average, and .50 large (Cohen, 1992). We used the inverse variance weight approach to perform the meta-analyses (Borenstein et al., 2009), and random effects models to pool effects size across samples based on the assumption that studies were not drawn from a single population but from a universe of populations, and hence heterogeneity was not due to sampling error alone (see Borenstein et al., 2009; Card, 2012).

Heterogeneity in effect sizes was assessed through the $Q$ statistic and the $I^{2}$ index. A significant $p$ value for the $Q$ test indicates that the total variance is not equal to the within-studies variance and thus between studies variance exists (Card, 2012). The $l^{2}$ statistic is a variance ratio indicating the percentage of variance that is due to betweenstudies heterogeneity. Values of 25, 50, and 75 are judged as low, moderate, and high, respectively (see Higgins \& Thompson, 2002).

Based on previous meta-analyses on risk factors for traditional dating violence (Hébert et al., 2017; Park \& Kim, 2018), we expected heterogeneity among our results and thus performed moderation analyses. Once again, we opted for random effects models based on our assumption that heterogeneity is not due to sampling error alone (Borenstein et al., 2009; Card, 2012). We performed meta-regressions using the method of moments because this method does not depend on any assumptions about the (normal) distribution of the random effects (Borenstein et al., 2009). We tested sex (\% of males), age (mean), and method quality (scores from 0 to 8 ) as moderators. We chose these demographic variables because they have been identified as moderators among primary studies (e.g., Bennett et al., 2011; Borrajo et al., 2015b; Smith et al., 2018).

Publication bias, i.e., the tendency to publish significant rather than non-significant results, was examined for each global effect using Egger et al.'s (1997) regression method. This method tests whether the funnel plot of the estimates (effect sizes vs. standard error) is symmetrical, as would be expected in the absence of publication bias. Additionally, the trim and fill procedure was used to assess adjusted effect sizes if the funnel plot presented asymmetry. 


\section{Results}

\section{Study Selection}

Figure 1. Flow Diagram.
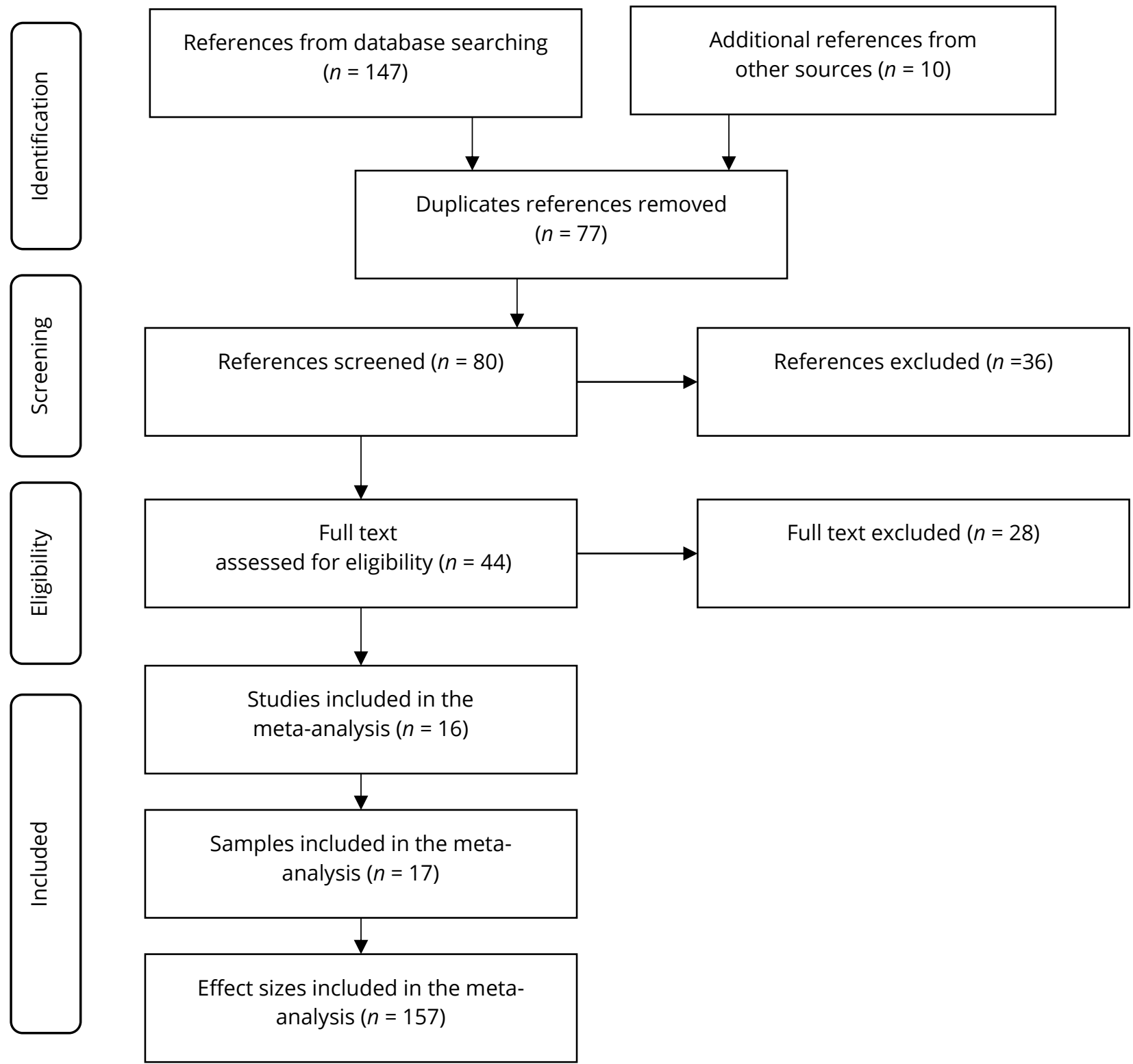

The flow diagram - Figure 1 - presents the selection process. Based on the combination of key words and Boolean characters to identify relevant manuscripts, the electronic databases searches resulted in 147 references. We added 10 more references through other sources, namely the hand search of relevant review articles and contact with authors in the field. Of these, 77 were duplicates and were removed. The titles and the abstracts of the remaining 80 references were then screened in order to decide if they could eventually meet our eligibility criteria. We excluded 36 references for the following main reasons: i) systematic or non-systematic reviews of the literature; ii) studies using only a qualitative methodology; (iii) articles in languages other than English, Spanish, or Portuguese. As a result, 44 full texts were retrieved and fully read to further assessed their eligibly. We excluded 28 references due to: i) studies presenting results on adults; iii) studies using an aggregated measure of cyber abuse including violence occurring both in an intimate dating relationship and in another context (e.g., with peers); ii) studies treating CDA as a predictor rather than an outcome; ii) no predictive analyses were performed. This process led to a final sample of 16 studies, 17 samples and 157 effect sizes included in the meta-analysis (see Figure 1 for the flowchart). 
Table 1. Descriptive Characteristics of 6 Eligible Studies About CDA Victimization and 6 Samples.

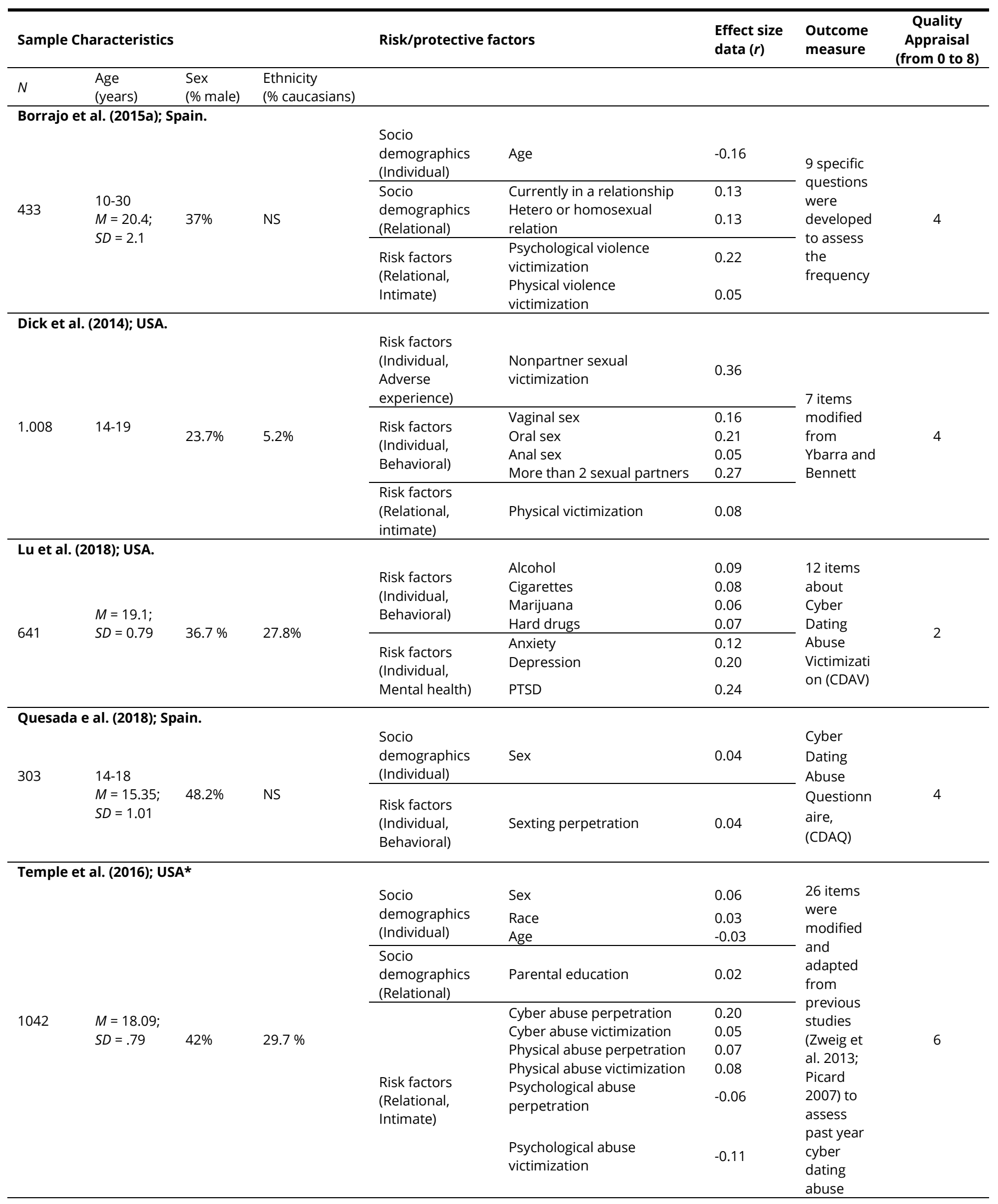

\section{Zweig et al. (2014); USA.}




\begin{tabular}{|c|c|c|c|}
\hline $\begin{array}{l}\text { Socio } \\
\text { demographics } \\
\text { (Relational) }\end{array}$ & $\begin{array}{l}\text { Living with both parents } \\
\text { LGBTQ relation }\end{array}$ & $\begin{array}{l}0.16 \\
0.33\end{array}$ & $\begin{array}{c}\text { cyber } \\
\text { dating } \\
\text { victimizati }\end{array}$ \\
\hline $\begin{array}{l}\text { Risk factors } \\
\text { (Individual, } \\
\text { Behavioral) }\end{array}$ & $\begin{array}{l}\text { Computer time } \\
\text { Cellphone time } \\
\text { Sexual relations } \\
\text { Alcohol } \\
\text { Marijuana } \\
\text { Serious drugs } \\
\text { Delinquency }\end{array}$ & $\begin{array}{l}0.11 \\
0.23 \\
0.72 \\
0.07 \\
-0.02 \\
0.06 \\
0.31\end{array}$ & on \\
\hline $\begin{array}{l}\text { Risk factors } \\
\text { (Individual, } \\
\text { Mental Health) }\end{array}$ & $\begin{array}{l}\text { Anger } \\
\text { Depressive } \\
\text { Anxiety }\end{array}$ & $\begin{array}{l}0.12 \\
0.11 \\
0.07\end{array}$ & \\
\hline $\begin{array}{l}\text { Risk factors } \\
\text { (Community, } \\
\text { School) }\end{array}$ & Bad grades in school & 0.23 & \\
\hline $\begin{array}{l}\text { Protective } \\
\text { factors } \\
\text { (Individual, } \\
\text { Behavioral) }\end{array}$ & Prosocial acts & -0.00 & \\
\hline $\begin{array}{l}\text { Protective } \\
\text { factors } \\
\text { (Relational, } \\
\text { Intimate) }\end{array}$ & $\begin{array}{l}\text { Positive relations with } \\
\text { partner }\end{array}$ & 0.06 & \\
\hline $\begin{array}{l}\text { Protective } \\
\text { factors } \\
\text { (Relational, } \\
\text { Family) }\end{array}$ & Parent closeness & -0.14 & \\
\hline $\begin{array}{l}\text { Protective } \\
\text { factors } \\
\text { (Community) }\end{array}$ & $\begin{array}{l}\text { School SES } \\
\text { Daily school }\end{array}$ & $\begin{array}{l}0.05 \\
0.11\end{array}$ & \\
\hline
\end{tabular}

Note. * The study investigated both outcomes - CDA victimization, and CDA perpetration. CDA perpetration is reported in Table 2.

Table 2. Descriptive Characteristics of 11 Eligible Studies About CDA Perpetration and 12 Samples.

\begin{tabular}{|c|c|c|c|c|c|c|c|c|}
\hline \multicolumn{4}{|c|}{ Sample Characteristics } & \multicolumn{2}{|c|}{ Risk/protective factors } & \multirow[t]{2}{*}{$\begin{array}{l}\text { Effect size } \\
\text { data }(r)\end{array}$} & \multirow[t]{2}{*}{$\begin{array}{l}\text { Outcome } \\
\text { measure }\end{array}$} & \multirow{2}{*}{$\begin{array}{c}\text { Quality } \\
\text { Appraisal } \\
\text { (from } 0 \text { to } 8 \text { ) }\end{array}$} \\
\hline N & $\begin{array}{l}\begin{array}{l}\text { Age } \\
\text { (years) }\end{array} \\
\text { year }\end{array}$ & $\begin{array}{l}\text { Sex } \\
\text { (\% male) }\end{array}$ & $\begin{array}{l}\text { Ethnicity } \\
\text { (\% caucasians) }\end{array}$ & & & & & \\
\hline \multicolumn{9}{|c|}{ Borrajo et al. (2015b); Spain. } \\
\hline \multirow{4}{*}{656} & \multirow{4}{*}{$\begin{array}{l}18-30 \\
M=22.58 \\
S D=4.8\end{array}$} & \multirow{4}{*}{$21 \%$} & \multirow{4}{*}{ NS } & \multirow{2}{*}{$\begin{array}{l}\text { Socio } \\
\text { demographics } \\
\text { (Individual) }\end{array}$} & Age & 0.04 & \multirow{4}{*}{$\begin{array}{l}\text { Online } \\
\text { Dating } \\
\text { Abuse } \\
\text { Questionn } \\
\text { aire }\end{array}$} & \multirow{4}{*}{3} \\
\hline & & & & & Sex & 0.04 & & \\
\hline & & & & $\begin{array}{l}\text { Risk factors } \\
\text { (Individual, } \\
\text { Psychosocial) }\end{array}$ & Myths about love & 0.04 & & \\
\hline & & & & $\begin{array}{l}\text { Risk factors } \\
\text { (Relational, } \\
\text { Intimate) }\end{array}$ & $\begin{array}{l}\text { Psychological violence } \\
\text { perpetration }\end{array}$ & 0.26 & & \\
\hline \multicolumn{9}{|c|}{ Deans \& Bhogal (2017); UK. } \\
\hline \multirow{6}{*}{189} & \multirow{6}{*}{$\begin{array}{l}M=19.2 \\
S D=1.94\end{array}$} & \multirow{6}{*}{$19.6 \%$} & \multirow{6}{*}{ NS } & $\begin{array}{l}\text { Socio } \\
\text { demographics } \\
\text { (Individual) }\end{array}$ & Sex & 0.07 & \multirow{6}{*}{$\begin{array}{l}\text { Cyber } \\
\text { Dating } \\
\text { Abuse } \\
\text { Questionn } \\
\text { aire } \\
\text { (CDAQ) }\end{array}$} & \multirow{6}{*}{3} \\
\hline & & & & \multirow{3}{*}{$\begin{array}{l}\text { Risk factors } \\
\text { (Individual, } \\
\text { Behavioral) }\end{array}$} & Physical aggression & 0.13 & & \\
\hline & & & & & Verbal aggression & 0.02 & & \\
\hline & & & & & Behavioral jealousy & 0.36 & & \\
\hline & & & & \multirow{2}{*}{$\begin{array}{l}\text { Risk factors } \\
\text { (Individual, } \\
\text { Psychosocial) }\end{array}$} & Cognitive jealousy & 0.16 & & \\
\hline & & & & & Emotional jealousy & 0.13 & & \\
\hline \multicolumn{9}{|c|}{ Van Ouytsel, Ponnet, \& Walrave (2017); Belgium. } \\
\hline \multirow{3}{*}{466} & \multirow{3}{*}{$\begin{array}{l}16-22 \\
M=17.99 \\
S D=0.92\end{array}$} & & & \multirow{3}{*}{$\begin{array}{l}\text { Socio } \\
\text { demographics } \\
\text { (Individual) }\end{array}$} & Age & 0.05 & \multirow{3}{*}{$\begin{array}{l}\text { Cyber } \\
\text { Dating } \\
\text { Abuse } \\
\text { Questionn }\end{array}$} & \multirow{3}{*}{3} \\
\hline & & $29 \%$ & NS & & Sex & 0.05 & & \\
\hline & & & & & Living with both parent & 0.05 & & \\
\hline
\end{tabular}




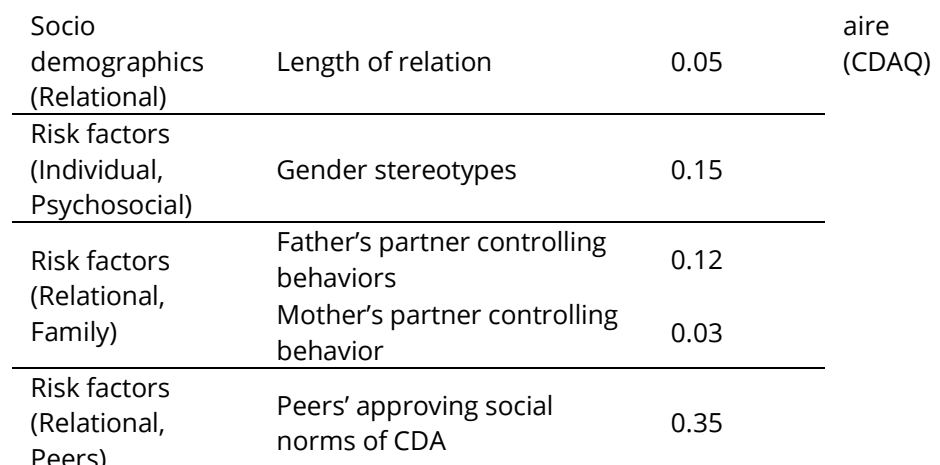

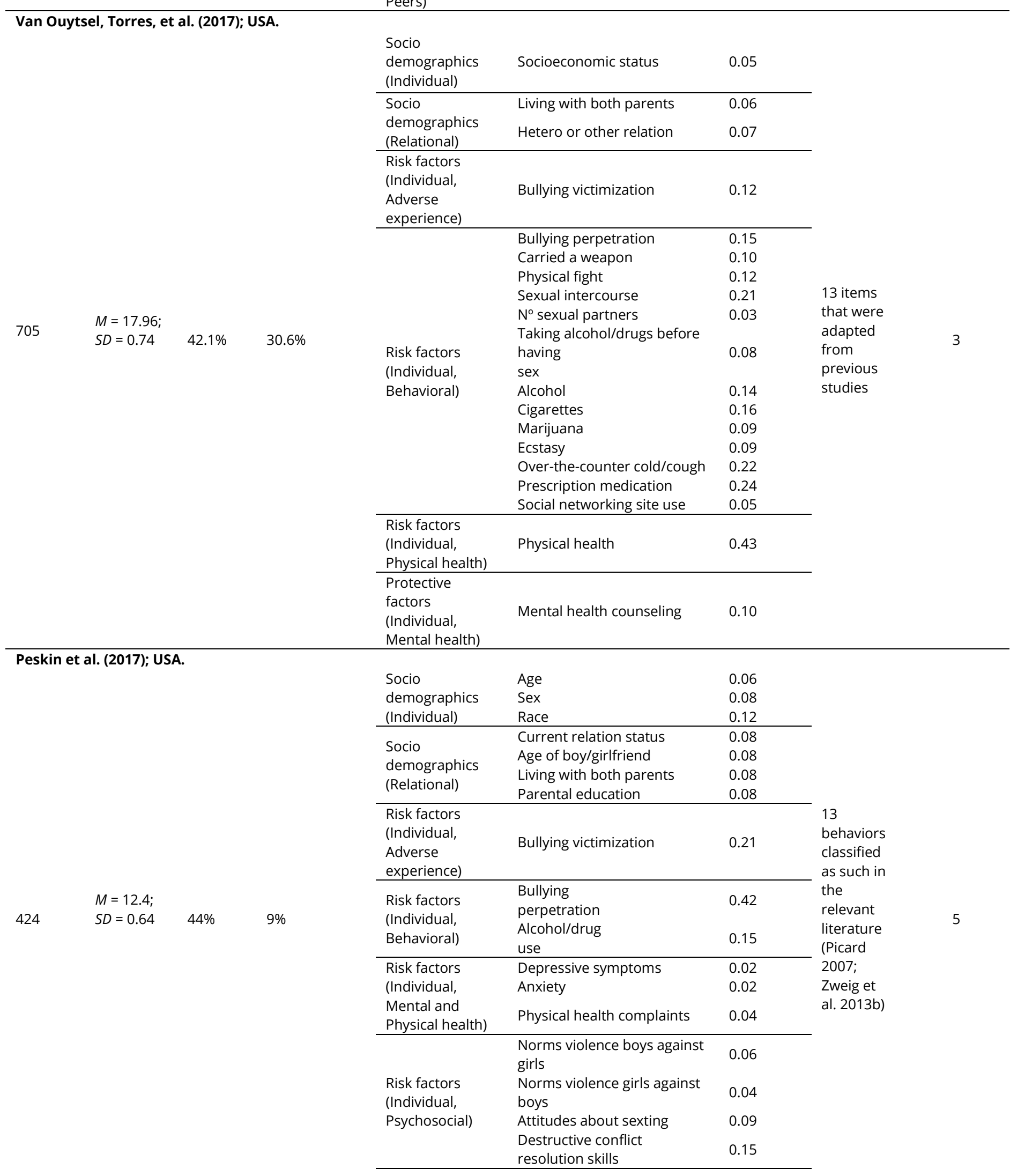




\begin{tabular}{|c|c|c|}
\hline $\begin{array}{l}\text { Risk factors } \\
\text { (Relational } \\
\text { Peers) }\end{array}$ & $\begin{array}{l}\text { Peer drug use } \\
\text { Peer } \\
\text { DA perpetration }\end{array}$ & $\begin{array}{l}0.09 \\
0.19\end{array}$ \\
\hline $\begin{array}{l}\text { Risk factors } \\
\text { (Community, } \\
\text { School) } \\
\end{array}$ & Bad grades in school & -0.05 \\
\hline $\begin{array}{l}\text { Protective } \\
\text { factors } \\
\text { (Individual, } \\
\text { Psychosocial) }\end{array}$ & $\begin{array}{l}\text { Positive coping strategies } \\
\text { Constructive conflict } \\
\text { resolution skills } \\
\text { Self-efficacy to resolve } \\
\text { conflict }\end{array}$ & $\begin{array}{l}0.08 \\
-0.04 \\
-0.12\end{array}$ \\
\hline $\begin{array}{l}\text { Protective } \\
\text { factors } \\
\text { (Relational, } \\
\text { Peers) }\end{array}$ & Social support from peers & -0.06 \\
\hline $\begin{array}{l}\text { Protective } \\
\text { factors } \\
\text { (Relational, } \\
\text { Family) }\end{array}$ & $\begin{array}{l}\text { Parental communication } \\
\text { Parental monitoring } \\
\text { Parent-child closeness }\end{array}$ & $\begin{array}{l}0.02 \\
-0.19 \\
-0.11\end{array}$ \\
\hline $\begin{array}{l}\text { Protective } \\
\text { factors } \\
\text { (Community) }\end{array}$ & $\begin{array}{l}\text { Social support from } \\
\text { community }\end{array}$ & -0.06 \\
\hline
\end{tabular}

\section{Reed et al. (2018); USA.}

Male sample.

$314 \quad M=16.40 \quad 100 \% \quad 75.6 \%$

Risk factors

(Individual,

Psychosocial)

Risk factors

(Relational,

Intimate)
Gender dating beliefs

Control CDA Victimization

Sexual CDA Victimization

Aggression CDA Victimization
0.19

0.43

3

socio

Female sample.

$M=16.40 \quad 0 \% \quad 75.6 \%$

Rivas \& Gimeno (2017); Spain.

$206 \quad \begin{aligned} & 13-20 \\ & M=15.88 ; \\ & S D=1.28\end{aligned}$

Smith-Darden et al. (2017); USA.

727

$49 \%$

\section{Socio}

$\begin{array}{llll}\text { demographics } & \text { Age } & 0.05 & \text { 36-item }\end{array}$

(Individual) measure

Risk factors adapted

Psychosocial) Tolman, \&

Risk factors $\quad$ Control CDA Victimization $\quad 0.50 \quad$ Ward,

(Relational, Sexual CDA Victimization 2016

Intimate) Aggression CDA Victimization

Risk factors

\begin{tabular}{ll} 
(Individual, $\quad 0.22$ \\
\hline
\end{tabular}

Mental Health)

\begin{tabular}{|c|c|c|c|}
\hline $\begin{array}{l}\text { Risk factors } \\
\text { (Relational, } \\
\text { Family) }\end{array}$ & Family conflict & 0.41 & $\begin{array}{l}\text { Scale of } \\
\text { Violence of } \\
\text { Couple in }\end{array}$ \\
\hline $\begin{array}{l}\text { Protective } \\
\text { factors } \\
\text { (Relational, } \\
\text { Family) }\end{array}$ & Family closeness & -0.17 & $\begin{array}{l}\text { the Social } \\
\text { Networks } \\
\text { in } \\
\text { Adolescent }\end{array}$ \\
\hline $\begin{array}{l}\text { Protective } \\
\text { factors } \\
\text { (Community) }\end{array}$ & $\begin{array}{l}\text { Involvement in class } \\
\text { School connectedness } \\
\text { Social support from teacher }\end{array}$ & $\begin{array}{l}-0.17 \\
-0.25 \\
-0.21\end{array}$ & \\
\hline
\end{tabular}

$M=16.6 ; \quad 49.8 \% \quad$ NS
$S D=1.06$

(Community)

Rodríguez-Domínguez et al. (2018); Spain.

$\begin{array}{llll}\text { (Individual, Gender dating beliefs } & 0.10 \quad \text { from Reed, } 4\end{array}$

Ward,

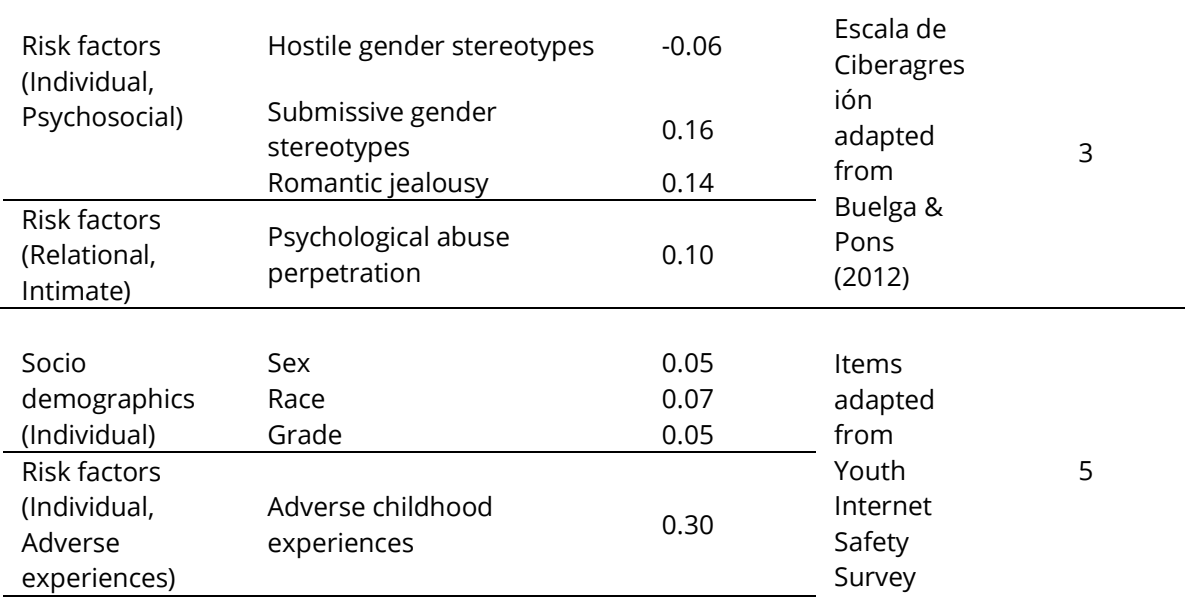




\begin{tabular}{|c|c|c|c|c|c|c|c|c|}
\hline & & & & $\begin{array}{l}\text { Risk factors } \\
\text { (Community) } \\
\end{array}$ & $\begin{array}{l}\text { School in area concentrated } \\
\text { disadvantage }\end{array}$ & -0.04 & & \\
\hline & & & & Protective & Family support & 0.02 & & \\
\hline & & & & factors & Parental involvement & -0.01 & & \\
\hline & & & & (Relational) & Peer support & -0.00 & & \\
\hline & & & & Protective & School connectedness & -0.04 & & \\
\hline & & & & factors & Community engagement & 0.04 & & \\
\hline & & & & (Community) & Neighborhood safety & -0.00 & & \\
\hline & al. (2016); U & A. * & & & & & & \\
\hline & & & & Socio & Sex & 0.04 & 26 items & \\
\hline & & & & demographics & Race & 0.04 & were & \\
\hline & & & & (Individual) & Age & 0.04 & modified & \\
\hline & & & & $\begin{array}{l}\text { Socio } \\
\text { demographics } \\
\text { (Relational) }\end{array}$ & Parental education & 0.04 & $\begin{array}{l}\text { and } \\
\text { adapted } \\
\text { from }\end{array}$ & \\
\hline & & & & & Cyber abuse perpetration & 0.27 & previous & \\
\hline 1042 & $M=18.09$ & & & & Cyber abuse victimization & -0.02 & studies & \\
\hline & $S D=0.79$ & $42 \%$ & $29.7 \%$ & & Physical abuse perpetration & -0.01 & (Zweig et & 5 \\
\hline & & & & & Physical abuse victimization & 0.07 & al. 2013; & \\
\hline & & & & $\begin{array}{l}\text { Risk factors } \\
\text { (Relational, } \\
\text { Intimate) }\end{array}$ & $\begin{array}{l}\text { Psychological abuse } \\
\text { perpetration }\end{array}$ & 0.03 & $\begin{array}{l}\text { Picard } \\
\text { 2007) to } \\
\text { assess }\end{array}$ & \\
\hline & & & & & $\begin{array}{l}\text { Psychological abuse } \\
\text { victimization }\end{array}$ & -0.03 & $\begin{array}{l}\text { past year } \\
\text { cyber } \\
\text { dating } \\
\text { abuse }\end{array}$ & \\
\hline Wrigh & 15); USA. & & & & & & & \\
\hline & & & & & Anxious partner attachment & 0.20 & Self- & \\
\hline 600 & $M=17.53$ & & & Risk factors & & & Partner- & \\
\hline 600 & $S D=0.51$ & $45.7 \%$ & $70.9 \%$ & (Relational, & & & Directed & 7 \\
\hline & & & & Intimate) & Avoidant partner attachment & 0.06 & Cyber & \\
\hline & & & & & & & $\begin{array}{l}\text { Aggression } \\
\text { (adapted) }\end{array}$ & \\
\hline
\end{tabular}

Note. * The study investigated both outcomes - CDA victimization, and CDA perpetration. CDA victimization is reported in Table 1.

Table 1 and Table 2 summarize the main descriptive characteristics of the 16 eligible studies and 17 samples, including a total of 12,760 participants. More than half of the studies was conducted in the United States of America (56.3\%), followed by Spain (31.3\%), and was published from 2017 to 2018 (62.5\%). The sample sizes ranged from 189 (Deans \& Bhogal, 2017) to 3,745 (Zweig et al., 2014), with a median of 750.6 participants. Only one study (Dick et al., 2014) use a clinical sample; all other samples were recruited from the community. With regard to participants' characteristics, the mean age ranged from $12.4(S D=0.64)$ (Temple et al., 2016) to 18.09 (SD $=0.79$ ) (Peskin et al., 2017). The majority of the studies (87.5\%) included results for female and male participants, one study examined female and male participants separately (Reed et al., 2018), and one sample was exclusively composed of males (Rodríguez et al., 2018). More than half of samples (58.8\%) included Caucasian participants, whose percentage ranged from 5.2\% (Dick et al., 2014) to 75.6\% (Reed et al., 2018).

As for the examined variables, in all the samples (100\%) one or more risk factors were examined (76.5\% individual, $64.7 \%$ relational and $11.8 \%$ community), $64.7 \%$ examined one or more sociodemographic factors (64.7\% individual and $35.3 \%$ relational), and $29.4 \%$ examined one or more protective factors ( $23.5 \%$ relational, $23.5 \%$ community and $11.8 \%$ individual). The main relational variables referred to intimate relations (68.6\%). Most of the studies (62.5\%) examined CDA perpetration as an outcome, 31.3\% examined CDA victimization, and one (Temple et al., 2016) analyzed both CDA victimization and perpetration using a prospective longitudinal study (the only study with this design in the present meta-analysis).

Most of the studies (56.3\%) used several items adapted and/or modified from others validated assessment measures of CDA, followed by the Cyber Dating Abuse Questionnaire (Borrajo, Gámez-Guadix, Pereda et al., 2015) (18.8\%). Other validated measures were used: The Online Dating Abuse Questionnaire ( $n=1$; Borrajo et al., 2015a), Scale of Violence of Couple in the Social Networks in Adolescents (E-Vpa) ( $n=1$; Rivas \& Gimeno, 2017), the Escala de Ciberagresión, adapted from Buelga and Pons (2012) $(n=1)$, and the Self-Reported Partner-Directed Cyber Aggression adapted from Linder et al. (2002) $(n=1)$. 
Finally, the quality appraisal ranged from a minimum of 2 (Lu et al., 2018) to 7 points (Wright, 2015), with average score of 4.4 .

\section{Meta-Analyses}

\section{Risk and Protective Factors of CDA Perpetration}

Table 3 presents the weighted mean effect sizes for the relations between sociodemographic, risk and protective factors with CDA perpetration, further divided in Individual (Adverse experiences, Behavioral, Mental and Physical Health, or Psychosocial), Relational (Intimate relations, Peer, or Family) and Community (School or Neighborhood)

Results indicated that no sociodemographic variable was significantly associated with CDA perpetration. This nonsignificant result held across the global upper case individual and relational categories, that included the combination of effect sizes of all variables of this nature, as well as for the lower case categories such as age, sex, characteristics of the intimate relation (e.g., duration, hetero vs homosexual), and parents' education level. The correlation coefficients were all below .10 or, when above this value (e.g., race/ethnicity), they presented wide confidence intervals.

Regarding risk factors for perpetrating CDA, Table 3 shows that both individual and relational risk factors held significant associations with CDA perpetration, with weighted mean correlation coefficients of .15 and .24 respectively. Analyzing the lower case categories of individual risk factors, adverse experiences (e.g., adverse childhood experiences, bullying victimization) and physical and mental health individual variables (e.g., physical health complaints, hostility, depression) revealed the highest weighted mean correlation coefficients, followed by behavioral variables (e.g., alcohol and/or drug use, sexual experiences, violence perpetration). Psychosocial variables (e.g., gender stereotypes, destructive resolution skills) sustained the smallest correlation coefficients among the individual risk factors. As for the lower case categories of relational risk factors, intimate relationship (e.g., offline violence perpetration and/or victimization) and peer variables (e.g., peer approving social norms of CDA, peer drug use) held significant weighted mean effect sizes of, respectively .24 and .25 , suggesting associations of a small to moderate magnitude. The global effect size for family relational risk factors (e.g., parents controlling behaviors of his/her partner, family conflict), albeit also of a small to moderate magnitude $(r=.25)$, did not reach statistical significance, as it presented a wide confidence interval. All the weighted mean correlations of the lower case categories were based, nonetheless, on a small pool of studies.

The weighted mean effect sizes for the relation between protective factors and CDA perpetration were, mainly, in the expected negative direction, as these factors would be related to a decrease in perpetration, but none were statistically significant ranging from -.09 to .02. However, these results were based, once again, on a small pool of studies.

Table 3 also shows the between study heterogeneity, assessed through the $\mathrm{Q}$ and $l^{2}$ index, and Egger's regression test of possible publication bias. As presented, the majority of the weighted mean effect sizes held significant and high levels of heterogeneity, as shown by the significant $Q$ tests and $l^{2}$ values superior to 75 . Among the individual, relational and community upper case categories, the only exceptions were community risk factors and individual protective factors, both computed from two independent studies. Also, six of the 16 lower case categories (e.g., parents' education, behavioral, peer protective factors), did not reveal heterogeneity in their global effect sizes. As for publication bias, results indicate no evidence of publication bias regarding the associations between sociodemographic variables, risk factors, and protective factors with CDA perpetration, with the exception of the association between this CDA outcome and individual risk factors. However, further exploring publication bias among this relation using the trim and fill method, we were able to conclude that no missing study was imputed and no adjustment of the individual risk factors-CDA perpetration correlation coefficient was needed. 
Table 3. Weighted Mean Effect Sizes for the Relationships Between Sociodemographic, Risk Factors, Protective Factors and Cyber Dating Abuse Perpetration.

\begin{tabular}{|c|c|c|c|c|c|c|c|c|c|}
\hline Associated variables & $k$ & $r$ & $95 \% \mathrm{Cl}$ & $p$ & $Q$ & $p$ & $1^{2}$ & $\begin{array}{l}\text { Egger's } \\
\text { test }\end{array}$ & $p$ \\
\hline \multicolumn{10}{|l|}{ Sociodemographic } \\
\hline Individual & 8 & .05 & $-.03, .14$ & .217 & $39.63^{* * *}$ & $<.001$ & 83.34 & 0.23 & .827 \\
\hline Sex & 6 & .00 & $-.10, .10$ & .978 & $29.78^{\star \star \star}$ & $<.001$ & 83.21 & 0.27 & .803 \\
\hline Age & 5 & .08 & $-.02, .17$ & .119 & $24.33^{* * *}$ & $<.001$ & 83.56 & 2.81 & .067 \\
\hline Race/Ethnicity & 3 & .11 & $-.03, .25$ & .123 & $6.79 *$ & .034 & 70.55 & 0.93 & .524 \\
\hline Others & 2 & .15 & $-.05, .33$ & .145 & $7.93 * *$ & .005 & 87.39 & & \\
\hline Relational & 4 & .05 & $-.04, .13$ & .253 & $8.17^{*}$ & .043 & 63.26 & 0.91 & .460 \\
\hline Intimate relations & 3 & .06 & $-.01, .13$ & .077 & 0.31 & .855 & 0.00 & 1.06 & .481 \\
\hline $\begin{array}{l}\text { Living with both } \\
\text { parents }\end{array}$ & 3 & .08 & $-.02, .19$ & .117 & 4.7 & .095 & 57.54 & 0.28 & .829 \\
\hline Parents' education & 2 & -.01 & $-.08, .05$ & .742 & 0.01 & .94 & 0.00 & & \\
\hline \multicolumn{10}{|l|}{ Risk factors } \\
\hline Individual & 10 & $.15^{\star \star \star}$ & $.09, .21$ & $<.001$ & $76.26^{\star \star \star}$ & $<.001$ & 88.20 & $4.49 * \star$ & .002 \\
\hline Adverse experiences & 3 & $.20 * *$ & $.08, .32$ & .001 & $6.38 *$ & .041 & 68.66 & 1.45 & .385 \\
\hline Behavioral & 3 & $.18^{* \star *}$ & $.10, .28$ & $<.001$ & 2.46 & .293 & 18.52 & 4.46 & .141 \\
\hline $\begin{array}{l}\text { Mental and physical } \\
\text { health }\end{array}$ & 4 & $.21 * \star$ & $.05, .36$ & .011 & $61.61 * * \star$ & $<.001$ & 95.13 & 3.32 & .080 \\
\hline Psychosocial & 7 & $.11 * \star \star$ & $.08, .15$ & $<.001$ & 5.39 & .494 & 0.00 & 0.44 & .681 \\
\hline Relational & 9 & $.24^{* \star *}$ & $.13, .36$ & $<.001$ & $163.45^{* * *}$ & $<.001$ & 95.11 & 0.37 & .722 \\
\hline Intimate relations & 6 & $.24^{\star *}$ & $.06, .40$ & .009 & $120.87^{* \star *}$ & $<.001$ & 95.86 & 1.15 & .314 \\
\hline Family & 2 & .25 & $-.09, .54$ & .152 & $38.60 * \star \star$ & $<.001$ & 97.41 & & \\
\hline Peers & 2 & $.25^{*}$ & $.03, .44$ & .023 & $12.15^{\star \star \star}$ & $<.001$ & 91.77 & & \\
\hline Community & 2 & -.04 & $-.13, .04$ & .305 & 0.00 & .970 & 0.00 & & \\
\hline \multicolumn{10}{|l|}{ Protective factors } \\
\hline Individual & 2 & .02 & $-.10, .14$ & .732 & 1.22 & .269 & 18.00 & & \\
\hline Relational & 3 & -.08 & $-.19, .04$ & .179 & $24.51 * * *$ & $<.001$ & 91.84 & 1.53 & .369 \\
\hline Family & 3 & -.08 & $-.20, .04$ & .186 & $23.03^{* * *}$ & $<.001$ & 91.31 & 1.22 & .436 \\
\hline Peers & 2 & -.00 & $-.03, .03$ & .779 & 0.33 & .568 & 0.00 & & \\
\hline Community & 3 & -.09 & $-.25, .09$ & .330 & $39.96 * * \star$ & $<.001$ & 95.00 & 0.79 & .575 \\
\hline
\end{tabular}

Notes. $k=$ number of effect sizes; $r=$ weighted mean correlation coefficient; $\mathrm{Cl}=$ confidence interval; $Q=$ test of homogeneity; $l^{2}=$ proportion between studies variability; Egger's test = Egger's regression test of publication bias (t-value)

${ }^{*} p<.05, * \star p<.01, * \star * p<.001$ (two-tailed).

\section{Risk and Protective Factors of CDA Victimization}

Weighted mean effect sizes for the relationship between sociodemographic variables (individual and relational), risk factors (individual and relational) and CDA victimization are reported in Table 4. We were unable to analyze community risk factors and protective factors for CDA victimization because only one study included these variables.

As shown, relational sociodemographic variables (i.e., intimate relations and family sociodemographics) were significantly associated with CDA victimization, with a weighted mean correlation of .08 . This significant correlation is mainly due to characteristics of the intimate relation $(r=.24)$, namely being of a homosexual nature and being currently in a relationship. No other sociodemographic variable reached statistical significance.

Individuals' risk factors for CDA victimization were, as a whole, significant, with a weighted mean effect size of .14. Among these, both behavioral (e.g., alcohol and drug use, sexual experiences, delinquency) and mental health 
variables (e.g., anxiety, depression, PTSD) were significantly associated with CDA victimization with correlation coefficients of small magnitude (.16 and .13, respectively). At last, despite the non-significant effect size of the upper case category Relational, intimate relations risk factors of CDA victimization (e.g., violence perpetration and/or victimization) did reach statistical significance although the effect size was of small magnitude $(r=.06)$. Results regarding CDA victimization were based on a small pool of studies.

Table 4. Weighted Mean Effect Sizes for the Relationships Between Sociodemographic, Risk Factors, Protective Factors and Cyber Dating Abuse Victimization.

\begin{tabular}{|c|c|c|c|c|c|c|c|c|c|}
\hline Associated variables & $k$ & $R$ & $95 \% \mathrm{Cl}$ & $p$ & $Q$ & $p$ & $P^{2}$ & Egger's test & $P$ \\
\hline \multicolumn{10}{|l|}{ Sociodemographic } \\
\hline Individual & 4 & .01 & $-.11, .13$ & .908 & $31.40 * * *$ & $<.001$ & 90.45 & 1.63 & .246 \\
\hline Sex & 3 & .14 & $-.06, .33$ & .167 & 46.18 & $<.001$ & 95.67 & 1.60 & .355 \\
\hline Age & 3 & -.02 & $-.18, .15$ & .860 & $42.28 * \star \star$ & $<.001$ & 95.38 & $24.13^{*}$ & .026 \\
\hline Race/Ethnicity & 2 & -.03 & $-.15, .08$ & .566 & $9.14^{* *}$ & .003 & 89.06 & & \\
\hline Relational & 3 & $.08^{* \star}$ & $.03, .13$ & .001 & 3.87 & .144 & 48.35 & 0.21 & .869 \\
\hline Intimate relations & 2 & $.24^{*}$ & $-.04,42$ & .021 & $16.63^{* * \star}$ & $<.001$ & 93.99 & & \\
\hline Family & 2 & -.07 & $-.25, .11$ & .434 & $22.85^{\star \star *}$ & $<.001$ & 95.62 & & \\
\hline \multicolumn{10}{|l|}{ Risk factors } \\
\hline Individual & 3 & $.14^{\star \star *}$ & $.09, .19$ & $<.001$ & $9.58^{* *}$ & .008 & 79.12 & 0.11 & .931 \\
\hline Behavioral & 3 & $.16^{\star \star}$ & $.06, .26$ & .003 & $26.76^{\star \star \star}$ & $<.001$ & 93.28 & 0.02 & .987 \\
\hline Mental health & 2 & $.13^{* *}$ & $.05, .21$ & .002 & $4.27^{*}$ & .039 & 76.55 & & \\
\hline Relational & 5 & .04 & $-.03, .11$ & .263 & $20.78^{\star \star}$ & $<.001$ & 80.75 & $4.42 *$ & .021 \\
\hline Intimate relations & 5 & $.06 * *$ & $.04, .09$ & $<.001$ & 3.24 & .518 & 0.00 & 0.47 & .669 \\
\hline
\end{tabular}

Notes. $k$ = number of effect sizes; $r$ = weighted mean correlation coefficient; $\mathrm{Cl}=$ confidence interval; $Q=$ test of homogeneity; $I^{2}=$ proportion between studies variability; Egger's test = Egger's regression test of publication bias (t-value)

${ }^{*} p<.05, * * p<.01, * \star * p<.001$ (two-tailed).

With regard to between-study heterogeneity, also presented in a Table 4, results indicate significant and high levels of heterogeneity among the weighted mean effect sizes, with the exception of those regarding sex, relational sociodemographics, and intimate relation risk factors. Finally, Table 4 presents Egger's regression tests of publication bias. As shown, only the weighted mean effect sizes of age and relational risk factors present evidence of possible publication bias. However, no missing study was imputed and no adjustments to these weighted mean effect sizes were made by using the trim and fill method.

\section{Moderation Analyses}

Moderation analyses were performed among the relations of individual and relational upper case categories of risk factors for CDA perpetration, as these effect sizes were computed from the largest number of studies $(n=10$ and $n=9$, respectively) and thus held greater statistical power. In addition, moderation effects were not tested among sociodemographic variables because the moderator variables were mainly of a sociodemographic nature. Results revealed that none of the tested moderators - sex, age, and method quality - held significant effects on the relations of individual (Sex: $b=0.00,95 \% \mathrm{Cl}[-0.00,0.00], p=.748$; Age: $b=0.01,95 \% \mathrm{Cl}[-0.01,0.03], p=.254$; Quality: $b=0.02,95 \% \mathrm{Cl}[-0.05,0.09], p=.634$ ) and relational risk factors (Sex: $b=-0.00,95 \% \mathrm{Cl}[-0.01,0.00], p=.665 ;$ Age: $b$ $=0.00,95 \% \mathrm{Cl}[-0.05,0.05], p=.936$; Quality: $b=-0.03,95 \% \mathrm{Cl}[-0.12,0.07], p=.574)$ with CDA perpetration; i.e., the relations "individual risk-CDA perpetration" and "relational risk-CDA perpetration" did not varying by the percentage of males, mean age of the participants or the methodological quality. Post-hoc power analyses indicated, however, that all the moderation analyses, with the exception the one testing age as a moderator of the individual-CDA perpetration relation (.89), were clearly under powered, with levels below Cohen's (1992) 80 conventional standard (ranging from .10 to .30), suggesting very low chances of detecting effects that genuinely exist. 


\section{Discussion}

We performed a meta-analysis of 16 studies and 17 independent samples, including 12,760 participants, in order to identify what risk and protective factors were associated to youth CDA perpetration and victimization. To the best of our knowledge, the current meta-analysis was the first to explore the overall risk and protective factors of youth CDA, in an attempt to provide a comprehensive picture of what factors most increase and decrease the probability of occurrence of CDA, and thus better inform strategies and policies to prevent this phenomenon. This should be the purpose of meta-analyses - systematize information of different academic work to support future research and practical interventions (cf., Hébert et al., 2017).

Our results confirmed those of past systematic reviews (e.g., Brown \& Hegarty, 2018; Caridade et al., 2019; Flach \& Deslandes, 2017; Gámez-Guadix et al., 2018): studies of CDA have focused on risk in detriment of protective factors and on individual and intimate relations variables in detriment of family, peer, and community factors. Specifically, with regard to the present meta-analysis, we found that $75 \%$ of studies analyzed risk factors for perpetrating CDA and $37.5 \%$ included risk factors for CDA victimization; the corresponding percentages for protective factors were $25 \%$ for CDA perpetration and $6.3 \%$ for CDA victimization. This is an indicator that protective factors are understudied, even though they constitute a promising line of research and of prevention efforts (Hébert et al., 2017). Likewise, individual variables were analyzed by $76.5 \%$ of the studies, while community factors were included only in $11.8 \%$. As for relational factors, these were explored by $64.7 \%$ of studies, but the most representative variables referred to intimate relations (47.1\%) in detriment of family (29.4\%) and peer (17.6\%) variables. The greater focus on individual risk factors may be explained by the fact that the inter-individual perspectives were one of the first theoretical approaches to emerge in the field of intimate violence (Caridade, 2018).

In addition, our results revealed that more studies focused on perpetration (62.5\%) than on victimization (31.3\%) and that only one study, a longitudinal study (Temple et al., 2016), analyzed these two outcomes. Searching for risk factors associated with victimization may lend support to perspectives of "blaming the victim" for their victimization and there has been reluctance to engage in such research (Siegel \& Williams, 2001). Research on traditional dating violence, for instance, has also focused primarily on risk factors for perpetrating physical dating violence, in attempt to understand how to prevent this type of abuse (Peskin et al., 2017). However, analyzing risk factors for victimization is useful for several reasons: i) to promote of a more holistic understanding of the problem, for example, describing the victims' greater psychological vulnerability to the occurrence of abuse (see Hydén, 1995), ii) to determine if the risk factors associated with CDA victimization are similar to those of traditional dating violence and other forms of abuse perpetrated through technologies (e.g., cyberbullying) (Gámez-Guadix et al., 2018); iii) to understand the reciprocity/mutuality of CDA, documented by past evidence (e.g., Leisring \& Giumetti, 2014; Morelli et al., 2017; Watkins et al., 2018; Reed et al., 2017), as well as to identify risk factors that increase the likelihood of engaging in both the perpetrator and victim roles; iv) and finally to develop prevention methods aimed at minimizing the consequences of CDA (Gracia-Leiva et al., 2019).

We believe that our results may have been affected by this greater focus by the included studies on certain risk, protective factors and outcomes, namely the small number analyzing protective factors, family and community predictors, as well as victimization as an outcome. Specifically, the different categories of protective factors for CDA perpetration - individual, relational, and community - did not reach statistical significance, although the majority were in the expected negative direction; protective factors were not analyzed for CDA victimization, as only one study (Zweig et al., 2014) included these variables. Family relational risk factors for CDA perpetration were not statistically significant and they were also absent for CDA victimization. This same trend was found for community risk factors: they were not associated with CDA perpetration and we were unable to analyze community risk factors for CDA victimization. These non-significant results were surprising and unexpected because they are inconsistent with what has been found in the past by primary data (e.g. Peskin et al., 2017; Rivas \& Gimeno, 2015; Smith-Darden et al., 2017; Van Ouytsel, Torres et al., 2017). Our results were also contrary to what has been found with regard to offline DV: parental and family variables are key risk factors of physical and psychological DV perpetration and victimization (cf. Taylor \& Xia, 2018). Gracia-Leiva et al. (2019), in their metaanalysis on DV, found that the social and community environment was more closely related to DV as opposed to individual factors. It is important to continue exploring these somewhat neglected risk and protective factors of 
youth CDA in order to determine if they are indeed unrelated to the phenomenon or if our conclusions result from the limited number of studies.

Nevertheless, we were able to draw some general and meaningful conclusions from our systematized empirical data. With regard to individual risk variables, results further showed that adverse experiences (e.g., adverse childhood experiences and bullying victimization), as well as physical and mental health individual variables, such as physical health complaints, hostility, and depression, revealed the highest weighted mean correlations, followed by behavioral variables (e.g., alcohol and/or drug use, sexual experiences, violence perpetration). Individual risk factors (mental health and behavioral) also held significant associations with CDA victimization, although of smaller magnitudes. The interaction of background factors (e.g., child abuse or others experiences of past victimization, aggressive personality traits, psychopathology) with situational factors (e.g., interpersonal conflict, substance use, relationship satisfaction, problem-solving skills, communication styles) has been identified by the background-situational theory as playing an important role in explaining DV (Riggs \& O'Leary, 1996). According to this approach, background factors promote individual aggressive patterns of behavior and situational factors increase conflict levels within the relationship; the risk of violence increases when more of these factors are present (Riggs \& O'Leary, 1989). Many of these individual-level risk factors associated with CDA, especially the behavioral ones, are similar to those arising in connection with the perpetration of traditional dating violence, reaffirming the importance of intervention efforts in this dimension (Peskin et al., 2017).

Intimate relation risk variables, such as offline violence perpetration and victimization, also were associated with both victimization and perpetration of CDA. This result further supports the background-situational theory (Riggs \& O'Leary, 1996), as these variables represent situational factors and the increased level of conflict in the relationship. In addition, the co-occurrence of CDA with offline dating violence has been consistently found in previous work (e.g., Cutbush et al., 2010; Hellevik, 2019; Hellevik \& Øverlien, 2016; Villora et al., 2019; Zapor et al., 2017; Zweig et al., 2013) and may reflect an escalation of violence between dating partners, with abuse being a form of self-defense or retaliation (Whitaker et al., 2007) and learned through the different social interactions, according to the social learning theory (Bandura, 1977, as cited by Villora et al., 2019). Also, several researchers (Korchmaros et al., 2013; Stonard et al., 2014; Van Ouytsel, Ponnet, Walrave, \& Temple., 2016) have suggested that CDA is often an extension of offline IPV perpetration or these are sometimes experienced as a continuum of violent behavior.

As previously exposed, we were not able to produced results on other relational risk factors of CDA victimization due to the small number of studies. We did however analyze family and peer risk factors of CDA perpetration, and concluded the first were not significantly related to CDA perpetration, but the second, peer risk factors (e.g., peer approving social norms of CDA, peer drug use), reached statistical significance. Once again, peer risk factors may represent situational factors of the background-situational theory (Riggs \& O'Leary, 1996), and mechanisms identified by the social learning theory may help understand this finding. Specifically, behaviors are learned through social interactions, and negative characteristics of the peer group can act as an unhealthy romantic relational model (Hertzog \& Rowley, 2014). It has also been argued that CDA perpetrators seek and associated with others who have similar attitudes towards behaviors (Van Ouytsel, Ponnet, \& Walrave, 2017). In a sociocultural viewpoint, the peer group norms have been established as a risk factor due to their influence on the activation of aggressiveness (e.g., anger, hostile attitudes, and aggressive behaviors) (cf. Aizpitarte et al., 2017). Furthermore, these perspectives explaining the influences of peers on adolescents and young adults' lives are not necessary mutually exclusive. However, our results should be interpreted with caution due to the small number of samples involved in the meta-analysis.

Finally, we concluded that individual sociodemographic variables were not associated neither to CDA perpetration nor CDA victimization. Respectively, being a girl or a boy, a Caucasian or belonging to another ethnicity, being of a younger or of an older age, are not risk of protective factors of perpetrating or of experiencing CDA. This conclusion is meaningful in the sense that it allows further clarification of the contradictory results revealed by previous primary studies (e.g., Deans \& Bhogal, 2017; Sánchez et al., 2015; Smith et al., 2018; Zweig et al., 2013) and better informs intervention programs in this field, stressing the importance of widespread and early prevention efforts, regardless of sociodemographic characteristics. 


\section{Moderation Analyses}

We found substantial between study heterogeneity across the majority of the estimated effect sizes, but our moderation analyses revealed that none of the tested moderators held significant effects on the relations between risk factors and CDA. These analyses were, however, limited in several ways. First, we tested three moderators sex, age, and methodological quality -, not including other variables, such as ethnicity, sampling frame, category of variables, due to the small number of samples per relation or due to their non-independence among the samples. Additionally, moderation analyses were performed only among the relations between individual and relational risk factors with CDA perpetration because the remaining relations presented a small number of samples or were of the same nature of our moderators (sociodemographics). Finally, post-hoc power analyses indicated that the majority of the moderation analyses were under powered, revealing very low chances of detecting effects that genuinely exist.

\section{Limitations and Future Research}

Apart from those regarding our moderation analyses, other limitations of the present meta-analysis should be considered when interpreting the results and should be taken into account by further research. First, our searches did not yield nonpublished studies (dissertations and research reports), and thus significant versus non-significant results might have been overrepresented in our meta-analysis (i.e., publication bias). Statistical tests of publication bias indicated, however, that there was no evidence of this bias among our results. Second, the search strategy in this review included commonly used search terms, not considering less frequent ones (e.g., "internet dating abuse" OR "social media dating abuse" OR "chat dating abuse"), an option that may be considered in future research seeking to replicate or expand this study. In addition, only one of the included studies used a longitudinal design, a more appropriate design (than the cross-sectional) given our aim of exploring risk and protective factors of youth CDA. Conducting longitudinal studies is essential to truly untangle the effects between risk, protective factors and consequences of CDA and thus should be a priority of future research. Fourth, the majority of the studies were conducted in the United States of America, restricting the generalizability of our conclusions. Given that risk and protective factors may operate differently according to the country in what the study was conducted (Jiménez et al., 2017), it is important to expand studies to other cultural contexts and realities. Fifth, experiences of CDA were based solely on the self-reports of the perpetrators and the victims and hence can present bias of this type of report (e.g., memory distortions). The use of official records (although also with intrinsic limitations) may lead to additional knowledge. Also, information on response rates and measures' reliability were often missing in the articles, thus coded as zero in the Cambridge Quality Checklist (Murray et al., 2009) and possibly resulting in poorer methodological quality scores. Seventh and as previously stated, the very small number of studies examining certain associated variables - protective factors, family, and community factors - as well as examining victimization as an outcome might have influenced our conclusions: we were not able to analyze some of these variables and others were not statically significant. These understudied variables should be a focus of future studies. Additionally, confidence intervals were mostly quite wide (i.e., > 0.10; see Campbell et al., 2009). Finally, and because CDA may be considered an extension of offline DV (Korchmaros et al., 2013; Van Ouytsel, Ponnet, \& Walrave., 2016) and these phenomena were indeed related in the present study, it is not clear if the risk and protective factors are unique to CDA or just part of a larger cluster of partner violence. It is important to assess and control this issue in future studies.

\section{Practical Implications}

The results from the present meta-analyze highlighted three important contexts of risk factors of youth CDAindividual, intimate relations and peers - that should be considered in the development of prevention and intervention efforts. Youth with certain individual background factors, such as past victimization experiences and mental health problems, are at increased risk of perpetrating and suffering from CDA, thus the early prevention of these background factors is highly recommended. These individual background factors may interact with situational risk factors, namely behavioral individual risk factors (e.g., alcohol and/or drug use), intimate relation risk variables (e.g., offline violence perpetration and victimization), and peer risk variables (e.g., peer drug use), highlighting the need to prevent and treat problem and high-risk behaviors. These results, in sum, stress the importance of promoting a healthy adolescent development in order to prevent victimization experiences, psychopathologies, and high-risk behaviors. 
Furthermore, the association between offline dating violence and CDA may indicate their co-occurrence and thus underlines the importance of continuous efforts to prevent offline dating violence, especially through a primary prevention approach. The results of our meta-analysis also suggest that the influence of peers as a risk factor should be taken into account when intervening in CDA perpetration. Taken together and in line with social learning mechanisms, it is essential to promote more adequate and healthy social models among both intimate and peer relations, in order to break the cycle of violence.

In addition, we found that individual sociodemographic factors, such as sex, age, and ethnicity, were unrelated to youth CDA and that sex and age were not significant moderators, which strongly indicates that prevention programs should not be directed to individuals with particular demographic characteristics. Another recent systematic review (Taylor \& Xia, 2018) concluded that men and women perpetrate CDA at equal rates, also suggesting the importance of not overlooking certain demographic characteristics in prevention efforts.

In sum, the present meta-analysis indicates that youth who have been victimized and have mental health problems, who use alcohol and/or drug, socialize with drug user peers, and who are involved in offline DV are a higher risk group, regardless of their sociodemographic characteristics, that could be targeted by prevention programs. Many existing programs have targeted individual and peer risk factors, which according to our conclusions must continue to be targets (La Rue et al., 2017). It is also important to develop programs based on innovative approaches, such as bystander approach (Storer et al., 2016), which has been identified as potentially powerful tools in preventing dating violence (Dos Santos \& Murta, 2019). This approach aims to allow youth to intervene and interrupt behaviors when witnessing situations that could lead abusive behaviors, concentrating on the role of peers and other social units.

Although the awareness-raising actions are important to prevent CDA, they are still insufficient to cope with the complexity of this phenomenon, requiring prevention efforts that extend in time, using a multidimensional and multi agent approach and considering skill-building components (De La Rue et al., 2017). Indeed, the results of the present meta-analysis lend support to ecological models, in the sense that they have highlighted factors from different contexts of the youth life which, in turn, stresses the importance of multifactorial and multi agent approaches in order to prevent CDA among youth.

\section{Authors' Note}

The views and opinions expressed in this article are those of the author and do not necessarily reflect the official policy or views of the Integration and Probation Penitentiary Services of France.

\section{References}

Studies included in the meta-analysis are identified by an asterisk.

Aizpitarte, A., Alonso-Arbiol, I., \& Van de Vijver, F. J. R. (2017). An explanatory model of dating violence risk factors in Spanish adolescents. Journal of Research on Adolescence, 27(4), 797-809. https://doi.org/10.1111/jora.12315

American Psychological Association (n.d.). Adolescence. In APA dictionary of psychology. Retrieved March 20, 2019, from https://dictionary.apa.org/adolescence

American Psychological Association (2002). Developing adolescents: A reference for professionals. American Psychological Society. https://www.apa.org/pi/families/resources/develop.pdf

APA Publications and Communications Board Working Group on Journal Article Reporting Standards. (2008). Reporting standards for research in psychology: Why do we need them? What might they be? American Psychologist, 63(9), 839-851. https://doi.org/10.1037/0003-066X.63.9.839 
Bennett, D. C., Guran, E. L., Ramos, M. C., \& Margolin, G. (2011). College students' electronic victimization in friendships and dating relationships: Anticipated distress and associations with risky behaviors. Violence and Victims, 26(4), 410-429. https://doi.org/10.1891/0886-6708.26.4.410

Borenstein, M., Hedges, L. V., Higgins, J. P. T., \& Rothstein, H. R. (2009). Introduction to meta-analysis. Wiley.

*Borrajo, E., Gámex-Guadix, M., \& Calvete, E. (2015a). Cyber dating abuse: Prevalence, context, and relationships with offline dating aggression. Psychological Reports, 116(2), 565-585.

https://doi.org/10.2466/21.16.PR0.116k22w4

*Borrajo, E., Gámex-Guadix, M., \& Calvete, E. (2015b). Justification beliefs of violence, myths about love and cyber dating abuse. Psicothema, 27(4), 327-333. https://doi.org/10.7334/psicothema2015.59

Borrajo, E., Gámex-Guadix, M., Pereda, N., \& Calvete, E. (2015). The development and validation of the cyber dating abuse questionnaire among young couples. Computers in Human Behavior, 48, 358-365.

https://doi.org/10.1016/j.chb.2015.01.063

Bronfenbrenner, U., \& Morris, P. A. (1998). The ecology of human developmental processes. In W. Damon \& N. Eisenberg (Eds.), The handbook of child psychology (3rd ed., pp. 993-1027). Wiley.

Brown, C., \& Hegarty, K. (2018). Digital dating abuse measures: A critical review. Aggression and Violent Behavior, 40, 44-59. https://doi.org/10.1016/j.avb.2018.03.003

Buelga, S., \& Pons, J. (2012). Agresiones entre adolescentes a través del teléfono móvil y de internet [Aggressions among teenagers through mobile phone and of internet]. Psychosocial Intervention, 21, 91-101. https://doi.org/10.5093/in2012v21n1a2

Burke, S. C., Wallen, M., Vail-Smith, K., \& Knox, D. (2011). Using technology to control intimate partners: An exploratory study of college undergraduates. Computers in Human Behavior, 27(3), 1162-1167.

https://doi.org/10.1016/j.chb.2010.12.010

Campbell, M. A., French, S., \& Gendreau, P. (2009). The prediction of violence in adult offenders: A meta-analytic comparison of instruments and methods of assessment. Criminal Justice and Behavior, 36(6), 567-590.

https://doi.org/10.1177/0093854809333610

Card, N. A. (2012). Applied meta-analysis for social science research. Guilford Press.

Caridade, S. (2018). Violência no namoro: Contextualização teórica e empírica [Dating violence: Theoretical and empirical contextualization]. In S. Neves \& A. Correia (Eds.), Violências no Namoro [Dating violence] (pp. 9-40). Edições ISMAI.

Caridade, S., Braga, T., \& Borrajo, E. (2019). Cyber dating abuse (CDA): Evidence from a systematic review. Journal of Aggression and Violent Behavior, 48, 152-168. https://doi.org/10.1016/j.avb.2019.08.018

Cohen, J. (1988). Statistical power analysis for the behavioral sciences (2nd ed). Erlbaum.

Cohen, J. (1992). A power primer. Psychological Bulletin, 112(1), 155-159. https://doi.org/10.1037/00332909.112.1.155

Cutbush, S., Ashley, O., Kan, M. L., \& Hall, D. M. (2010, November 6-10). Electronic aggression among adolescent dating partners: Demographic correlates and associations with academic performance and other types of violence [Paper presentation]. American Public Health Association 138th Annual Meeting \& Expo, Denver, CO, United States. https://apha.confex.com/apha/138am/webprogram/Paper229575.html 
Dardis, C. M., Dixon, K. J., Edwards, K. M., \& Turchik, J. A. (2015). An examination of the factors related to dating violence perpetration among young men and women and associated theoretical explanations: A review of the literature. Trauma, Violence, \& Abuse, 16(2), 136-152. https://doi.org/10.1177/1524838013517559

*Deans, H., \& Bhogal, M. S. (2017). Perpetrating cyber dating abuse: A brief report on the role of aggression, romantic jealousy and gender. Current Psychology, 38(5), 1077-1082. https://doi.org/10.1007/s12144-017-9715-4

De La Rue, L., Polanin, J. R., Espelage, D. L., \& Pigott, T. D. (2017). A meta-analysis of school-based interventions aimed to prevent or reduce violence in teen dating relationships. Review of Educational Research, 87(1), 7-34. https://doi.org/10.3102/0034654316632061

Dos Santos, K. B., \& Murta, S. G. (2019). Peers as agents of dating violence prevention: Feasibility analysis of an intervention. Trends in Psychology, 27(3), 631-646. https://doi.org/10.9788/TP2019.3-03

*Dick, R. N., McCauley, H. L., Jones, K. A., Tancredi, D. J., Goldstein, S., Blackburn, S., Monasterio, E., James, L., Silverman, J. G., \& Miller, E. (2014). Cyber dating abuse among teens using school-based health centers. Pediatrics, 134(6), e1560-e1567. https://doi.org/10.1542/peds.2014-0537

Egger, M., Smith, G. D., Schneider, M., \& Minder, C. E. (1997). Bias in meta-analysis detected by a simple graphical test. British Medical Journal, 315(7109), 629-634. https://doi.org/10.1136/bmj.316.7129.469

Farrington, D. P., Ttofi, M. M., \& Piquero, A. R. (2016). Risk, promotive, and protective factors in youth offending: Results from the Cambridge study in delinquent development. Journal of Criminal Justice, 45, 63-70.

https://doi.org/10.1016/j.jcrimjus.2016.02.014

Flach, R. M. D., \& Deslandes, S. F. (2017). Abuso digital nos relacionamentos afetivo-sexuais: uma análise bibliográfica [Cyber dating abuse in affective and sexual relationships: a literature review]. Reports in Public Health 33(7), Article e00138516. http://cadernos.ensp.fiocruz.br/csp/artigo/162/abuso-digital-nosrelacionamentos-afetivo-sexuais-uma-analise-bibliografica

Gámez-Guadix, M., Borrajo, E., \& Calvete, E. (2018). Partner abuse, control and violence through Internet and smartphones: Characteristics, evaluation and prevention. Psychologist Papers, 39(3), 218-227.

https://doi.org/10.23923/pap.psicol2018.2874

Gámez-Guadix, M., Villa-George, F., \& Calvete, E. (2014). Psychometric properties of the Cyberbullying Questionnaire (CBQ) among Mexican adolescents. Violence and Victims, 29(2), 232-247.

https://doi.org/10.1891/0886-6708.VV-D-12-00163R1

Gracia-Leiva, M., Puente-Martínez, A., Ubillos-Landa, S., \& Páez-Rovira, D. (2019). Dating violence (DV): A systematic meta-analysis review. Annals of Psychology, 35(2), 300-313.

https://doi.org/10.6018/analesps.35.2.333101

Hébert, M., Daspe, M.-E., Lapierre, A., Godbout, N., Blais, M., Fernet, M. \& Lavoie, F. (2017). A meta-analysis of risk and protective factors for dating violence victimization: The role of family and peer interpersonal context. Trauma, Violence, \& Abuse, 20(4), 574-590. https://doi.org/10.1177/1524838017725336

Hellevik, P. M. (2019). Teenagers' personal accounts of experiences with digital intimate partner violence and abuse. Computers in Human Behavior, 92, 178-187. https://doi.org/10.1016/j.chb.2018.11.019

Hellevik, P., \& Øverlien, C. (2016). Teenage intimate partner violence: Factors associated with victimization among Norwegian youths. Scandinavian Journal of Public Health, 44(7), 702-708.

https://doi.org/10.1177/1403494816657264 
Hertzog, J. L., \& Rowley, R. L. (2014). My beliefs of my peers' beliefs: Exploring the gendered nature of social norms in adolescent romantic relationships. Journal of Interpersonal Violence, 29(2), 348-368.

https://doi.org/10.1177/0886260513505145

Higgins, J. P. T., \& Thompson, S. G. (2002). Quantifying heterogeneity in a meta-analysis. Statistics in Medicine, 21(11), 1539-1558. https://doi.org/10.1002/sim.1186

Hyde, J. S. (2005). The gender similarities hypothesis. American Psychologist, 60(6), 581-592.

https://doi.org/10.1037/0003-066X.60.6.581

Hydén, M. (1995). Verbal aggression as a prehistory of woman battering. Journal of Family Violence, 10(1), 55-71. https://doi.org/10.1007/BF02110537

Jiménez, S., Muñoz-Fernández, V., Lucio López, L. A., \& Ortega-Ruíz, R. (2017). Ciberagresión en parejas adolescentes: Un estudio transcultural España-México [Cyber-aggression in adolescent couples: A cross-cultural study Spain-Mexico]. Revista Mexicana de Psicología, 34(1), 46-54.

Korchmaros, J. D., Ybarra, M. L., Langhinrichsen-Rohling, J., boyd, d., \& Lenhart,A. (2013). Perpetration of teen dating violence in a networked society. Cyberpsychology, Behavior, and Social Networking, 16(8), 561-567.

https://doi.org/10.1089/cyber.2012.0627

Leisring, P., \& Giumetti, G. (2014). Sticks and stones may break my bones, but abusive text messages also hurt: Development and validation of the cyber psychological abuse scale. Partner Abuse, 5(3), 323-341.

https://doi.org/10.1891/1946-6560.5.3.323

Linder, J. R., Crick, N. R., \& Collins, W. A. (2002). Relational aggression and victimization in young adults' romantic relationships: Associations with perceptions of parent, peer, and romantic relationship quality. Social Development, 11(1), 69-86. https://doi.org/10.1111/1467-9507.00187

Lipsey, M. W., \& Wilson, D. B. (2001). Practical meta-analysis. Sage.

*Lu, Y., Van Ouytsel, J., Walrave, M., Ponnet, K., \& Temple, J. R. (2018). Cross-sectional and temporal associations between cyber dating abuse victimization and mental health and substance use outcomes. Journal of Adolescence, 65, 1-5. https://doi.org/10.1016/j.adolescence.2018.02.009

Moher, D., Liberati, A., Tetzlaff, J., \& Altman, D. G. (2009). Preferred reporting items for systematic reviews and meta-analyses: The PRISMA statement. PLoS Medicine, 6(7), Article e1000097.

https://doi.org/10.1371/journal.pmed.1000097

Morelli, M., Bianchi, D., Chirumbolo, A., \& Baiocco, R. (2017). The cyber dating violence inventory. Validation of a new scale for online perpetration and victimization among dating partners. European Journal of Developmental Psychology, 15(4), 464-471. https://doi.org/10.1080/17405629.2017.1305885

Murray, J., Farrington, D. P., \& Eisner, M. P. (2009). Drawing conclusions about causes from systematic reviews of risk factors: The Cambridge Quality Checklists. Journal of Experimental Criminology, 5(1), 1-23.

https://doi.org/10.1007/s11292-008-9066-0

Park, S., \& Kim, S.-H. (2018). The power of family and community factors in predicting dating violence: A metaanalysis. Aggression and Violent Behavior, 40, 19-28. https://doi.org/10.1016/j.avb.2018.03.002

Perry, D. G., \& Pauletti, R. E. (2011). Gender and adolescent development. Journal of Research on Adolescence, 21(1), 61-74. https://doi.org/10.1111/j.1532-7795.2010.00715.x

*Peskin, M. F., Marklam, C. M., Shegog, R., Temple, J. R., Baumler, E. R., Addy, R. C., Hernandez, B., Cuccaro, P., Gabay, E. K., Thiel, M., \& Tortolero Emery, S. (2017). Prevalence and correlates of the perpetration of cyber dating 
abuse among early adolescentes. Journal of Youth and Adolescence, 46(2), 358-375.

https://doi.org/10.1007/s10964-016-0568-1

Peterson, R. A., \& Brown, S. P. (2005). On the use of beta coefficients in meta-analysis. Journal of Applied Psychology, 90(1), 175-181. https://doi.org/10.1037/0021-9010.90.1.175

Pujazon-Zazik, M., \& Park, M. J. (2010). To tweet, or not to tweet: Gender differences and potential positive and negative health outcomes of adolescents' social Internet use. American Journal of Men's Health, 4(1), 77-85. https://doi.org/10.1177/1557988309360819

*Quesada, S., Fernández-González, L., \& Calvete, E. (2018). El sexteo (sexting) en la adolescência: Frecuencia y asociación com la victimización de ciberacoso y violência en el noviazgo [The sex (sexting) in adolescence: Frequency and association with the victimization of cyber crime and violence in the news]. Behavioral Psychology, 26(2), 225-242.

Reed, L. A., Tolman, R. M., \& Ward, L. M. (2016). Snooping and sexting: Digital media as a context for dating aggression and abuse among college students. Violence Against Women, 22(13), 1556-1576.

https://doi.org/10.1177/1077801216630143

Reed, L. A., Tolman, R. M., \& Ward, L. M. (2017). Gender matters: Experiences and consequences of digital dating abuse victimization in adolescent dating relationships. Journal of Adolescence, 59, 79-89.

https://doi.org/10.1016/j.adolescence.2017.05.015

*Reed, L. A., Ward, L. M., Tolman, R. M., Lippman, J. R., \&. Seabrook, R. C. (2018). The association between stereotypical gender and dating beliefs and digital dating abuse perpetration in adolescent dating relationships. Journal of Interpersonal Violence. Advance online publication. https://doi.org/10.1177/0886260518801933

Reyns, B. W., Henson, B., \& Fisher, B. S. (2011). Being pursued online: Applying cyberlifestyle-routine activities theory to cyberstalking victimization. Criminal Justice and Behavior, 38(11), 1149-1169.

https://doi.org/10.1177/0093854811421448

Riggs, D. S., \& O'Leary, D. K. (1989). A theoretical model of courtship aggression. Violence in dating relationships: Emerging social issues. Praeger.

Riggs, D. S., \& O'Leary, D. K. (1996). Aggression between heterosexual dating partners: An examination of a causal model of courtship aggression. Journal of Interpersonal Violence, 11(4), 519-540.

https://doi.org/10.1177/088626096011004005

*Rivas, M. M., \& Gimeno, C. M. (2017). Violencia de pareja virtual y ajuste psicossocial en la adolescência desde la perspetiva de género [Online teen dating violence and psychosocial adjustment from a gender perspective]. International Journal of Developmental and Educational Psychology, 2(1), 115-124.

https://doi.org/10.17060/ijodaep.2017.n1.v2.924

*Rodríguez-Domínguez, C., Segura, M. D., \& Martínez-Pecino, R. (2018). Ciberagresores en el noviazgo adolescente y su relación con la violencia psicológica, el sexismo y los celos [Cyber aggressor in dating relationships and its relation with psychological violence, sexism, and jealousy]. Health and Addictions, 18(1), 1727. http://dx.doi.org/10.21134/haaj.v18i1.329

Rosenthal, R. (1994). Parametric measures of effect size. In H. Cooper \& L. V. Hedges (Eds.), The handbook of research synthesis (pp. 231-244). Russell Sage Foundation.

Sánchez, V., Muñoz-Fernández, N., \& Ortega-Ruíz, R. (2015). “Cyber dating Q_A": An instrument to assess the quality of adolescent dating relationships in social networks. Computers in Human Behavior, 48, 78-86.

https://doi.org/10.1016/j.chb.2015.01.006 
Seiffge-Krenke, I., \& Connolly, J. (2010). Adolescent romantic relationships across the globe: Involvement, conflict management, and linkages to parents and peer relationships. International Journal of Behavioral Development, 34(2), 97-97. https://doi.org/10.1177/0165025409360289

Siegel, J. A. \& Williams, L. M. (2001). Risk factors for violent victimization of women: A prospective study, summary. National Criminal Justice Reference Service. https://www.ncjrs.gov/pdffiles1/nij/grants/189160.pdf

Smith, K., Cénat, J. M., Lapierre, A., Dion, J., Hébert, M., \& Côté, K. (2018). Cyber dating violence: Prevalence and correlates among high school students from small urban areas in Quebec. Journal of Affective Disorders, 234, 220223. https://doi.org/10.1016/j.jad.2018.02.043

*Smith-Darden, J. P., Kernsmith, P. D., Victor, B. G., \& Lathrop, R. A. (2017). Electronic displays of aggression in teen dating relationships: Does the social ecology matter? Computers in Human Behavior, 67, 33-40.

https://doi.org/10.1016/j.chb.2016.10.015

Stanley, T. D., \& Jarrell, S. B. (1989). Meta-regression analysis: A quantitative method of literature surveys. Journal of Economic Surveys, 3(2), 161-170. https://doi.org/10.1111/j.1467-6419.1989.tb00064.x

Stonard, K. E., Bowen, E., Lawrence, T. R., \& Price, S. A. (2014). The relevance of technology to the nature, prevalence and impact of adolescent dating violence and abuse: A research synthesis. Aggression and Violent Behavior, 19(4), 390-417. https://doi.org/10.1016/j.avb.2014.06.005

Storer, H. L., Casey, E., \& Herrenkohl, T. (2016). Efficacy of bystander programs to prevent dating abuse among youth and young adults: A review of the literature. Trauma, Violence, \& Abuse, 17(3), 256-269.

https://doi.org/10.1177/1524838015584361

Taylor, S., \& Xia, Y. (2018). Cyber partner abuse: A systematic review. Violence and Victims, 33(6), 983-1011. https://doi.org/10.1891/0886-6708.33.6.983

*Temple, J. R., Choi, H. J., Brem, M., Wolford-Clevenger, C., Stuart, G. L., Flescher Peskin, M., \& Elmquist, J. (2016). The temporal association between traditional and cyber dating abuse among adolescents. Journal of Youth and Adolescence, 45(2), 340-349. https://doi.org/10.1007/s10964-015-0380-3

Van Ouytsel, J., Ponnet, K., \& Walrave, M. (2016). Cyber dating abuse victimization among secondary school students from a lifestyle-routine activities theory perspective. Journal of Interpersonal Violence, 33(17), 2767-2776. https://doi.org/10.1177/0886260516629390

*Van Ouytsel, J., Ponnet, K., \& Walrave, M. (2017). Cyber dating abuse: Investigating digital monitoring behaviors among adolescents from a social learning perspective. Journal of Interpersonal Violence. Advance online publication. https://doi.org/10.1177/0886260517719538

Van Ouytsel, J., Ponnet, K., Walrave, M., \& Temple, J. R. (2016). Adolescent cyber dating abuse victimization and its associations with substance use, and sexual behaviors. Public Health, 135, 147-151.

https://doi.org/10.1016/j.puhe.2016.02.011

*Van Ouytsel, J., Torres, E., Choi, H. J., Ponnet, K., Walrave, M., \& Temple, J. R. (2017). The associations between substance use, sexual behaviors, bullying, deviant behaviors, health, and cyber dating abuse perpetration. The Journal of School Nursing, 33(2), 116-122. https://doi.org/10.1177/1059840516683229

Villora, B., Yubero, S., \& Navarro, R. (2019). Cyber dating abuse and masculine gender norms in a sample of male adults. Future Internet, 11(4), Article 84. https://doi.org/10.3390/fi11040084

Watkins, L. E., Maldonado, R. C., \& DiLillo, D. (2018). The Cyber Aggression in Relationships Scale: A new multidimensional measure of technology-based intimate partner aggression. Assessment, 25(5), 608-626.

https://doi.org/10.1177/1073191116665696 
Whitaker, D. J., Haileyesus, T., Swahn, M., \& Saltzman, L. S. (2007). Differences in frequency of violence and reported injury between relationships with reciprocal and nonreciprocal intimate partner violence. American Journal of Public Health, 97(5), 941-947. https://doi.org/10.2105/AJPH.2005.079020

*Wright, M. F. (2015). Cyber aggression within adolescents' romantic relationships: Linkages to parental and partner attachment. Journal Youth Adolescence, 44(1), 37-47. https://doi.org/10.1007/s10964-014-0147-2

Zapor, H., Wolford-Clevenger, C., Elmquist, J., Febres, J., Shorey, R. C., Brasfield, H., Leisring, P., \& Stuart, G. (2017). Psychological aggression committed through technology: A study with dating college student. Partner Abuse, 8(2), 127-145. https://doi.org/10.1891/1946-6560.8.2.127

Zweig, J. M., Dank, M., Yahner, \& Lachman, P. (2013). The rate of cyber dating abuse among teens and how it relates to other forms of teen dating violence. Journal of Youth and Adolescence, 42(7), 1063-1077.

https://doi.org/10.1007/s10964-013-9922-8

*Zweig, J. M., Lachman, P., Yahner, J. \& Dank, M. (2014). Correlates of cyber dating abuse among teens. Journal of Youth and Adolescence, 43(8), 1306-1321. https://doi.org/10.1007/s10964-013-0047-x 


\section{Correspondence to:}

Sónia Caridade

Faculty of Human and Social Sciences

Praça 9 de Abril, 349

4249-004 Oporto

Portugal

Tel.: +351225071300

Fax: +351225508269

Email: soniac(at)ufp.edu.pt

Editorial record: First submission received on December 28, 2019. Revisions received on March 27, 2020 and May 28, 2020. Accepted for publication on June 1, 2020.

Editor in charge: Lenka Dedkova

\section{About Authors}

Sónia Caridade, Ph.D. in Forensic and Legal Psychology, is a Professor in the Faculty of Human and Social Sciences at Fernando Pessoa University, Oporto, Portugal. Her research interests include dating violence and cyber dating abuse, delinquency and behavior problems and their risk and protective factors.

Teresa Braga, Ph.D. in Forensic and Legal Psychology (University of Minho, Portugal), is a Psychologist at the Integration and Probation Penitentiary Services of Aisne, France. Her research interests include delinquency and offending and their risk and protective factors. 